\title{
AGR-1 Data Qualification Report
}

\author{
Mike Abbott \\ Larry Hull \\ Binh Pham \\ Mitch Plummer
}

March 2010

The INL is a

U.S. Department of Energy

National Laboratory

operated by

Battelle Energy Alliance

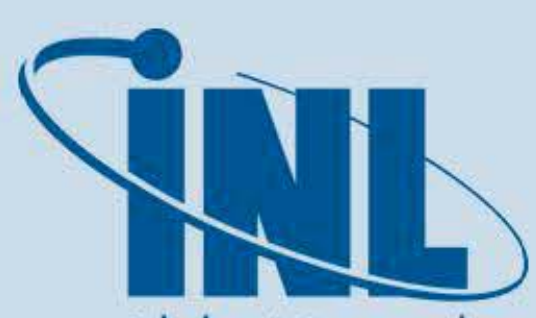

Idaho National Laboratory

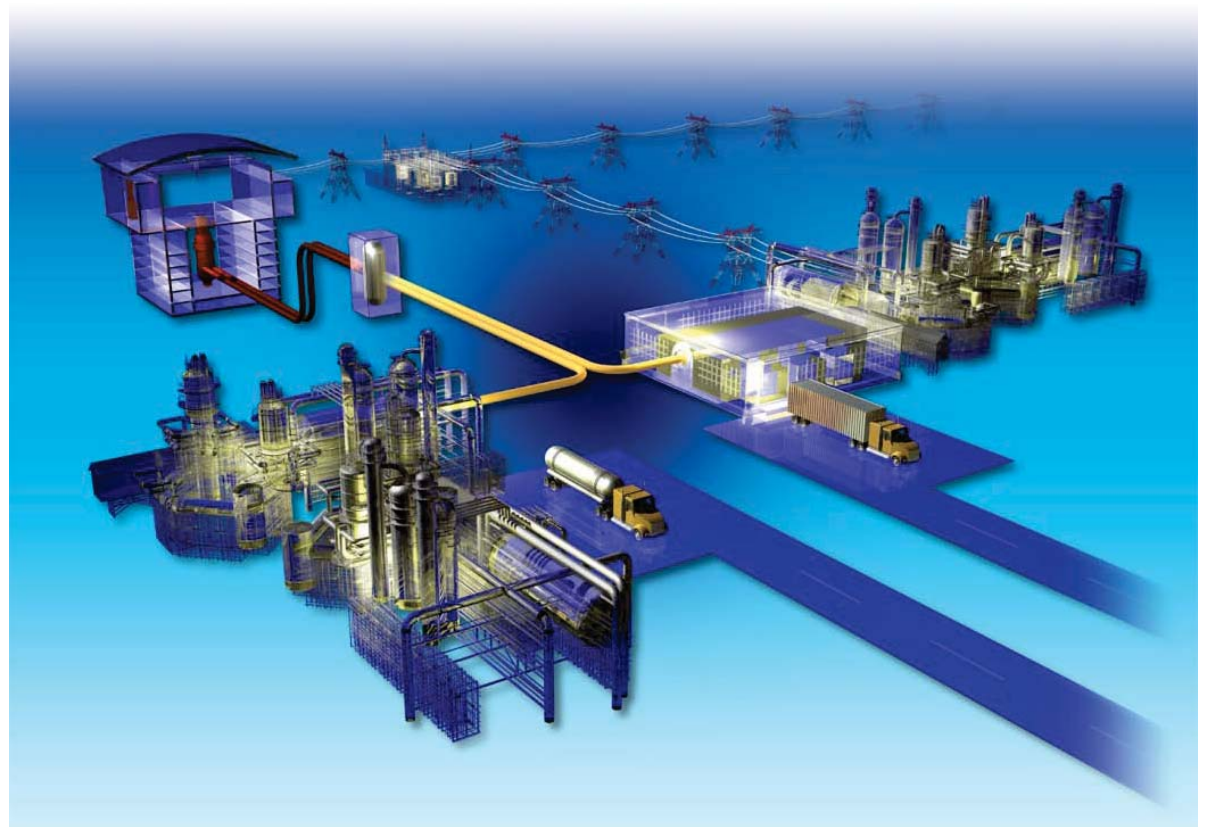




\section{DISCLAIMER}

This information was prepared as an account of work sponsored by an agency of the U.S. Government. Neither the U.S. Government nor any agency thereof, nor any of their employees, makes any warranty, expressed or implied, or assumes any legal liability or responsibility for the accuracy, completeness, or usefulness, of any information, apparatus, product, or process disclosed, or represents that its use would not infringe privately owned rights. References herein to any specific commercial product, process, or service by trade name, trade mark, manufacturer, or otherwise, does not necessarily constitute or imply its endorsement, recommendation, or favoring by the U.S. Government or any agency thereof. The views and opinions of authors expressed herein do not necessarily state or reflect those of the U.S. Government or any agency thereof. 


\title{
AGR-1 Data Qualification Report
}

\author{
Mike Abbott \\ Larry Hull \\ Binh Pham \\ Mitch Plummer
}

March 2010

\begin{abstract}
Idaho National Laboratory
Next Generation Nuclear Plant Project

Idaho Falls, Idaho 83415
\end{abstract}

http://www.inl.gov

Prepared for the

U.S. Department of Energy

Office of Nuclear Energy

Under DOE Idaho Operations Office

Contract DE-AC07-05ID14517 



\section{Next Generation Nuclear Plant Project}

\section{AGR-1 Data Qualification Report}

INL/EXT-10-17943

March 2010

Approved by:

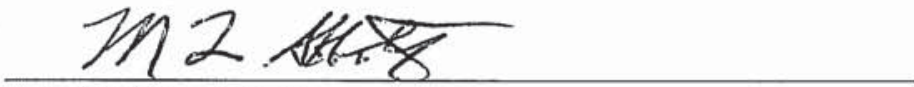

Michael L. Abbott

NDMAS AGR Data Stream Lead

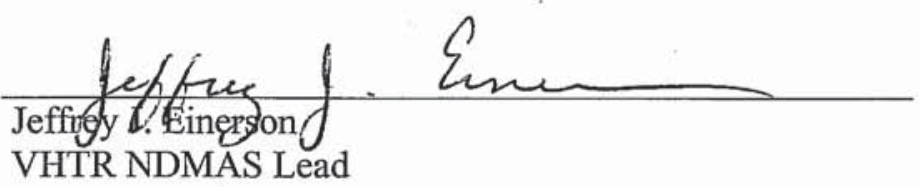

Alave Ven

Diane V. Croson

VHTR TDO Deputy Director

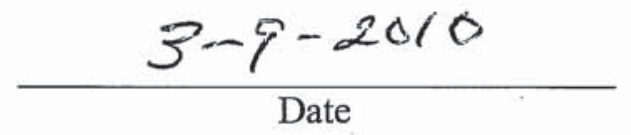

$3-9-2010$ Date

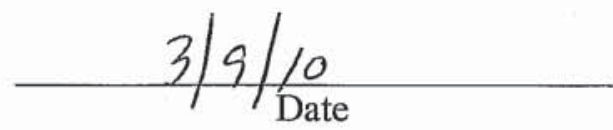




\section{ABSTRACT}

Projects for the very high temperature reactor (VHTR) Technology Development Office program provide data in support of Nuclear Regulatory Commission licensing of the VHTR. Fuel and materials to be used in the reactor are tested and characterized to quantify performance in high temperature and high fluence environments. The VHTR program has established the NGNP Data Management and Analysis System (NDMAS) to ensure that VHTR data are qualified for use, stored in a readily accessible electronic form, and analyzed to extract useful results.

This document focuses on the first NDMAS objective. It describes the data streams associated with the first Advanced Gas Reactor experiment (AGR-1), the processing of these data within NDMAS, and reports the qualification status of the data. Data qualification activities within NDMAS for specific types of data are determined by the data qualification category assigned by the data generator. They include: (1) capture testing, to confirm that the data stored within NDMAS are identical to the raw data supplied, (2) accuracy testing to confirm that the data are an accurate representation of the system or object being measured, and (3) documentation that the data were collected under an NQA-1 or equivalent Quality Assurance program. A summary of the NDMAS database processing and qualification status for the five AGR-1 data streams reported in this document is as follows:

1. Fuel fabrication data. All data have been processed into the NDMAS database and qualified (1,819 records).

2. Fuel irradiation data. Data from all 13 AGR-1 reactor cycles have been processed into the NDMAS database and tested (11,496,872 records). Of these, $85 \%$ have been qualified and $15 \%$ have failed NDMAS accuracy testing.

3. Fission Product Monitoring System data. Reprocessed (January 2010) data from all 13 AGR-1 reactor cycles have been processed into the database and capture tested (652,752 records). Final qualification of these data will be recorded after Quality Assurance approval of an Engineering Calculations and Analysis Report currently in review.

4. Advanced Test Reactor Operating Conditions Data. Data for all AGR-1 cycles have been stored and capture tested. These data, which come from outside the VHTR program, are assumed to be qualified by Advanced Test Reactor quality control procedures.

5. Neutronics and Thermal Simulation Data. NDMAS processing is in progress. Qualification of these data will be recorded after receipt of approved modeling reports produced by the data generators. 


\section{CONTENTS}

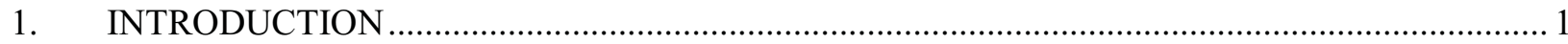

2. OVERVIEW OF NDMAS DATA QUALIFICATION ......................................................... 1

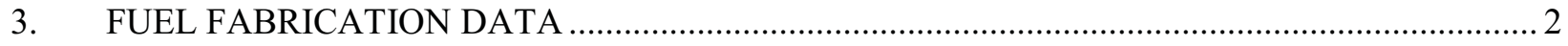

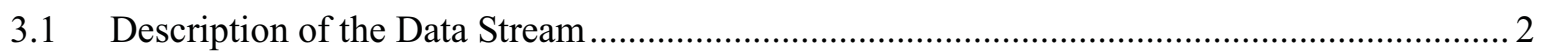

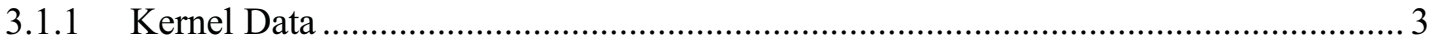

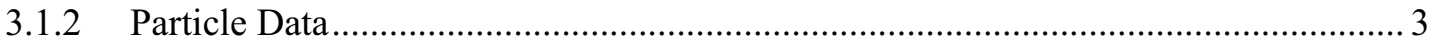

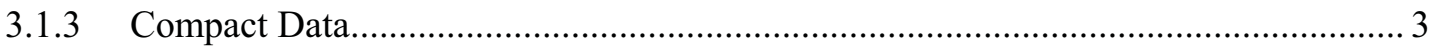

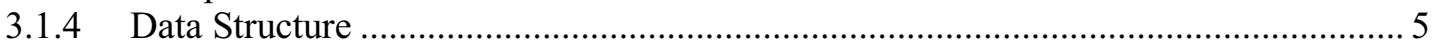

3.2 Fuel Fabrication Data Processing within NDMAS ........................................................ 7

3.3 Description of Fuel Fabrication Data Qualification ........................................................ 7

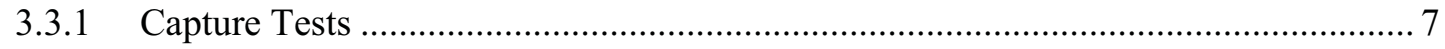

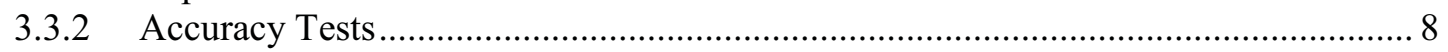

3.4 Verify Fuel Fabrication Data QA Documentation ............................................................ 8

3.5 Fuel Fabrication Data Qualification Status ....................................................................... 8

3.6 Fuel Fabrication Data Problems and Resolution................................................................ 9

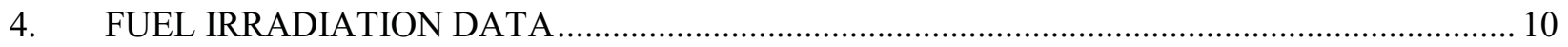

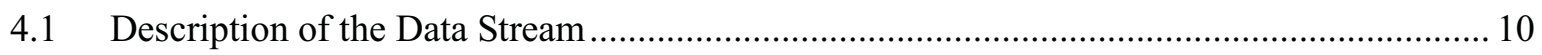

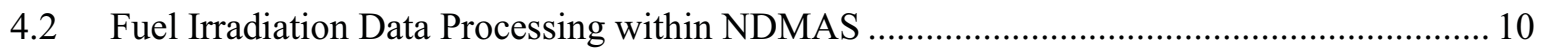

4.3 Description of Fuel Irradiation Data Qualification Tests..................................................... 12

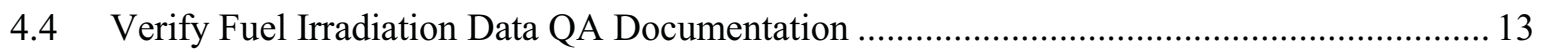

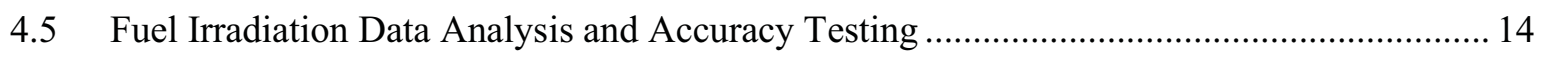

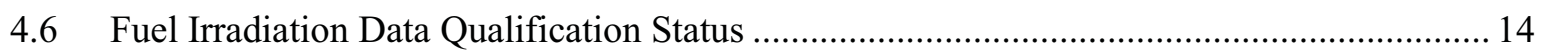

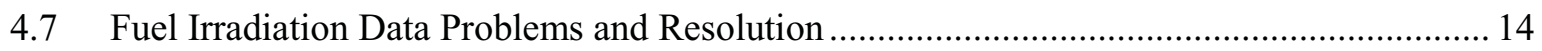

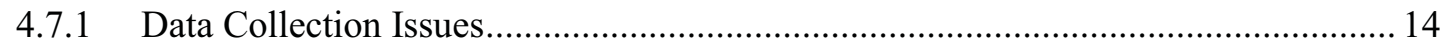

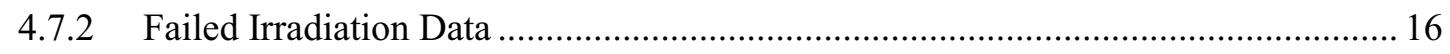

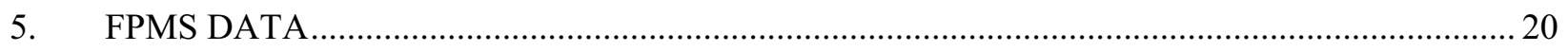

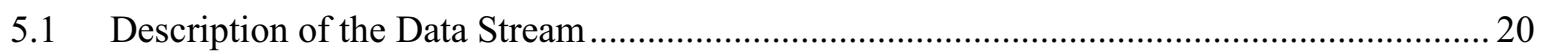

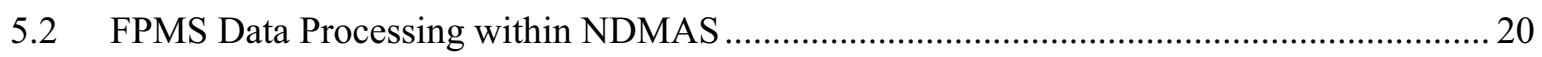

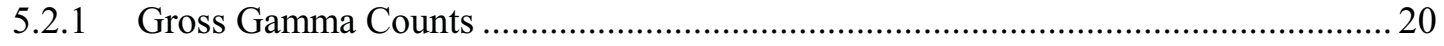

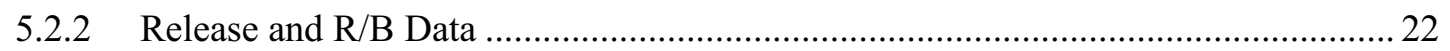

5.3 Description of FPMS Data Qualification Tests................................................................. 22

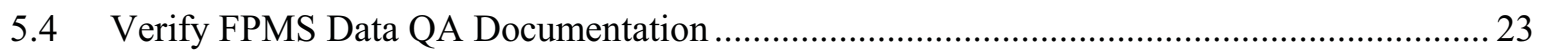

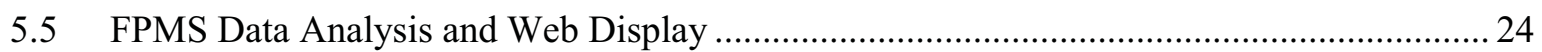

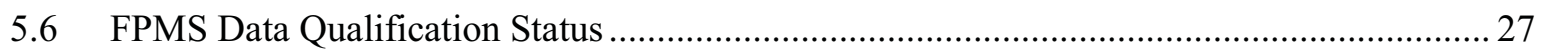

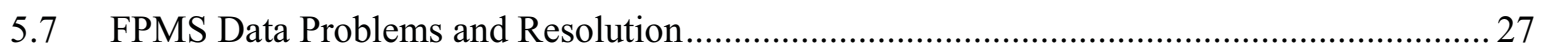

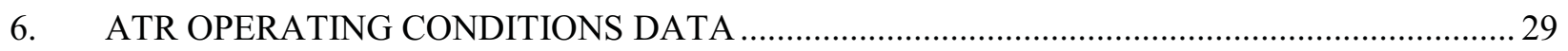

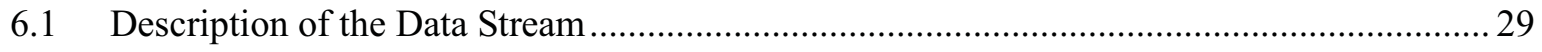

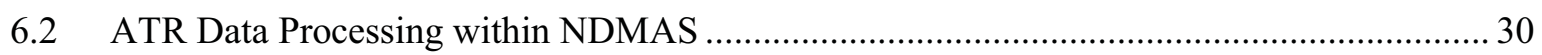




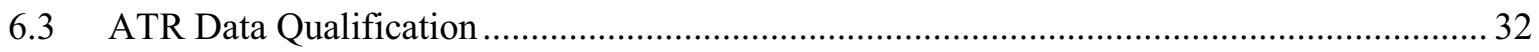

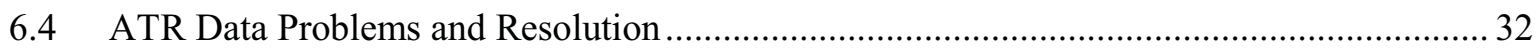

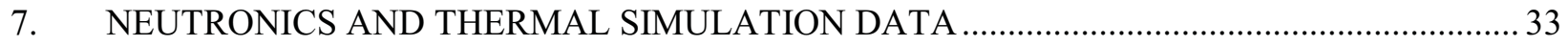

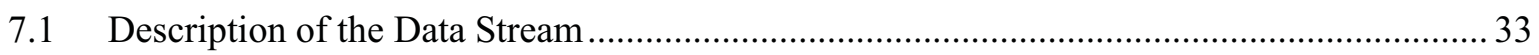

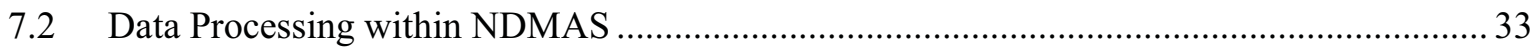

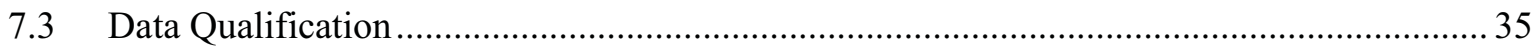

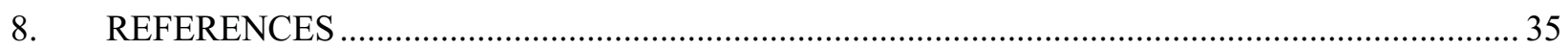

Appendix A—NDMAS Database Join for Compacts and Modeling Cells ............................................ 38

\section{FIGURES}

Figure 1. Fuel fabrication data component assembly tree structure..................................................... 5

Figure 2. AGR-1 thermocouple descriptions and location information................................................. 11

Figure 3. Raw AGR-1 irradiation data and data processing steps. ..................................................... 11

Figure 4. Gas moisture content data (log scale) from AGR-1 that failed accuracy tests

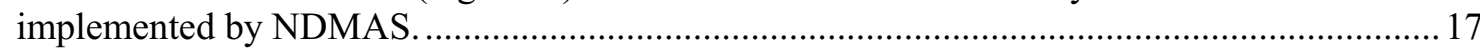

Figure 5. Gas pressure data (log scale) from AGR-1 that failed accuracy tests implemented by

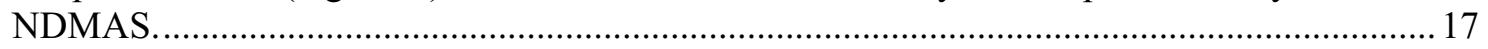

Figure 6. Gas flow data from AGR-1 that failed accuracy tests implemented by NDMAS..................... 18

Figure 7. Failed thermocouple temperature data from AGR-1, based on information provided to NDMAS by the NGNP technical leads. .......................................................................... 19

Figure 8. SAS processing of FPMS data (radionuclide release rates and R/B ratios). ........................... 22

Figure 9. Example of an R/B data plot available on the NDMAS Web portal........................................24

Figure 10. Example of release and R/B box plots available on the NDMAS Web portal. The boxes represent the $75 \%$ and $25 \%$ quartiles, with the center line representing the median R/B for each ATR cycle by capsule

Figure 11. Example of FPMS box plots on the NDMAS Web portal showing Kr-85m R/B

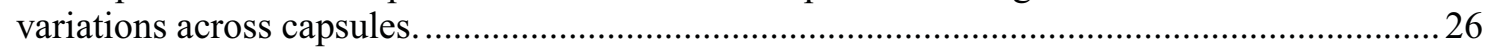

Figure 12. Example of [Log] R/B data distribution analysis available on the NDMAS Web portal.........26

Figure 13. Raw ATR operating conditions data and processing steps................................................ 31

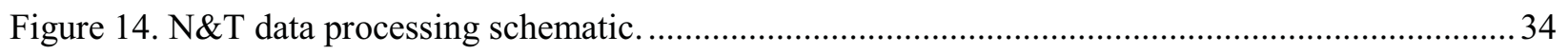

Figure 15. NDMAS links the modeling cell number to compact stack, loading levels, and lot names (shown here for top of stack 2)

\section{TABLES}

Table 1. Properties and specifications for AGR-1 fuel kernels (Lot G73D-20-69302)............................ 3

Table 2. Properties and specifications for AGR-1 coated particle composites......................................... 4

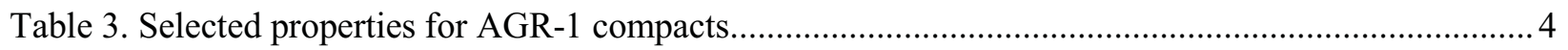


Table 4. Component types and names for AGR-1 fuel data. .............................................................. 5

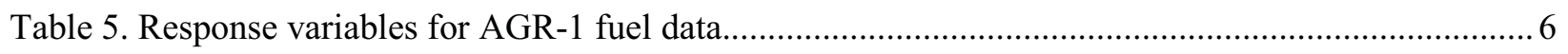

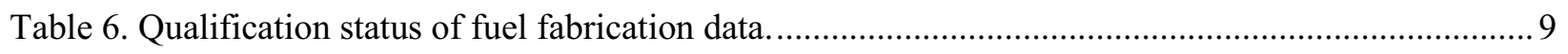

Table 7. Measurement variables in the fuel irradiation data stream. ...................................................... 10

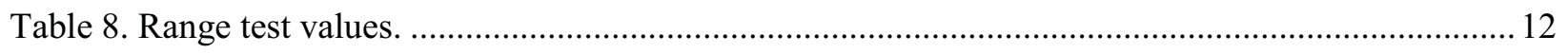

Table 9. Qualification status of fuel irradiation monitoring data received as of August 1, 2009............. 15

Table 10. Daylight savings time changes recorded in AGR-1 records, with corresponding dates and times for the correct time of change and time using pre-2007 policy............................... 16

Table 11. Chronology of thermocouple failures during AGR-1, based on NDMAS-assigned failure dates. Information provided to NDMAS is shown in the "AGR TC Table" column. Because thermocouple failure does not always coincide with complete failure of the instrument to respond to reactor conditions, the date of that failure, if it occurred, is shown as the 'Failure to respond' date.....

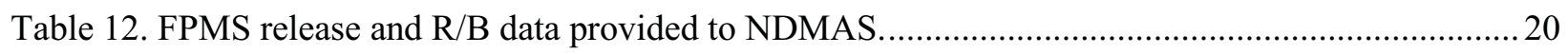

Table 13. Current NDMAS database status of AGR-1 FPMS release and R/B data..............................2 27

Table 14. ATR operating conditions variables stored in NDMAS. ......................................................29

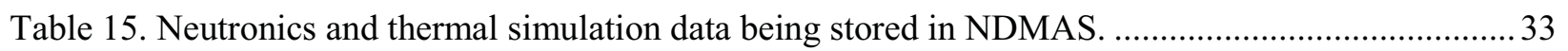




\section{ACRONYMS}

AGR Advanced Gas Reactor

ASME American Society of Mechanical Engineers

ATR Advanced Test Reactor

BWXT BWX Technologies

DST daylight-saving time

ECAR Engineering Calculations and Analysis Report

FPMS Fission Product Monitoring System

HPGe High Purity Germanium (detector)

INL Idaho National Laboratory

IPyC inner pyrolytic carbon layer (fuel kernel coating layer)

MDT Mountain Daylight Time

MST Mountain Standard Time

NDMAS NGNP Data Management and Analysis System

NGNP Next Generation Nuclear Plant

NQA Nuclear Quality Assurance

ORNL Oak Ridge National Laboratory

OPyC Outer pyrocarbon (fuel kernel coating layer)

PDF Portable Document Format

QA Quality Assurance

QAPP Quality Assurance Program Plan

$\mathrm{R} / \mathrm{B} \quad$ release-to-birthrate [ratio]

$\mathrm{SiC} \quad$ Silicon carbide (fuel kernel coating layer)

SQL Structured Query Language

TDO Technology Development Office

TFR Technical and Functional Requirements

VHTR Very High Temperature Reactor (program) and very high temperature gas-cooled reactor 


\section{AGR-1 Data Qualification Report}

\section{INTRODUCTION}

This report provides the final FY 2009 status of Advanced Gas Reactor AGR-1 irradiation experiment data qualification as performed by the NGNP Data Management and Analysis System (NDMAS). AGR-1 is the first in a series of eight planned irradiation experiments for the AGR Fuel Development and Qualification Program, which supports development of the very high temperature gas-cooled reactor (VHTR) under the Next Generation Nuclear Plant (NGNP) Project. Irradiation of the AGR-1 test train was conducted over 13 Advanced Test Reactor (ATR) cycles beginning with Cycle 138B on December 23, 2006, and ending with Cycle 145A on November 6, 2009.

This report gives the data qualification status of the three primary AGR-1 data streams, or general types of data, currently being collected and processed by NDMAS from the AGR Fuel Development and Qualification Project: Fuel Fabrication, Fuel Irradiation, and Fission Product Monitoring System (FPMS) data. It also reports on processing of two additional data streams in NDMAS, ATR Operating Conditions and Neutronics and Thermal Simulation data, which are not qualified by NDMAS. Data qualification of graphite characterization data collected under the Graphite Technology Development Project is reported in a separate status report (Hull 2009). Post-irradiation examination of AGR-1 fuel will commence in FY 2010 and continue for several months. Qualification of post-irradiation examination data will begin as soon as data become available.

\section{OVERVIEW OF NDMAS DATA QUALIFICATION}

NDMAS was developed to provide a single controlled repository for all NGNP data, documentation of and assistance in data qualification, advanced data analysis, and Web access of the data. A detailed discussion of the NDMAS structure and the data qualification requirements performed within NDMAS is given in the Very High Temperature Reactor Program Data Management and Analysis Plan (INL 2009a).

Data qualification is the act of reviewing, inspecting, testing, checking, or otherwise verifying and documenting whether data conform to specified requirements, as defined by the data users and the performing organization. Depending upon the data stream, data qualification may be performed by one or more entities, including independent technical reviewers, Quality Assurance (QA), data review committees, and testing performed within NDMAS. This process also considers whether the data were collected within a Nuclear Quality Assurance NQA-1 (American Society of Mechanical Engineers [ASME] 2000) or equivalent approved QA program. Data qualification within NDMAS is documented as one of three qualification states:

1. Qualified. Data that are independently verified to meet requirements, including documentation of formal certification reviews for the Fuel Fabrication and FPMS data streams and NDMAS accuracy testing for the Fuel Irradiation data stream. All qualified data must have been collected within an NQA-1 or equivalent approved QA program.

2. Trend. Data collected within an NQA-1 or equivalent approved QA program where the qualification process identifies minor flaws or gaps in meeting data requirements, although the data are still considered useful by the program. Trend data captured into NDMAS are tested to verify capture, and may be subjected to some accuracy testing.

3. Failed. The data do not meet specified requirements. This may be for a number of reasons, including inadequate data collection methods, instrument failure or drift, or poor accuracy.

There may also be data types collected within the program that are for information only. These data are not qualified because specific data qualification requirements cannot be defined or independent verification is not needed or desired. One example of this data type is the gross gamma FPMS data, which 
are binary detector output files used only as an early indicator of potential fuel particle failures (see Section 5). These data are processed by NDMAS for display on the web and are then archived or stored in their native file format for possible future use.

As part of the qualification process, capture testing is performed for all data uploaded into the NDMAS database. Capture testing includes automated checks to verify there are no obvious data processing errors in the source files (e.g., date/time chronology checks) and that the data stored within NDMAS are identical to the source data provided to NDMAS.

The primary component of data qualification is verifying data accuracy. For the Fuel Fabrication and FPMS data streams, program-specific data approval certifications or approved Engineering Calculations and Analysis Reports (ECARs) are used to verify and document data accuracy within NDMAS. For the Fuel Irradiation data stream, where no formal data certification exists, NDMAS accuracy testing is performed to identify anomalies in the data that may represent instrument drift or failure. For AGR-1, this accuracy testing included visual examination of the data, simple automated range tests (e.g., thermocouple temperature range of 0 to $1,300^{\circ} \mathrm{C}$ ), and more detailed analyses that use statistical analysis of past behavior to determine whether current instrument readings are reliable. Anomalies identified by these tests are then examined with input from the technical leads and resolved to determine whether the anomalies represent (1) instrument failures or other errors that disqualify ("fail") the data or (2) values that are unusual but reliable thereby resulting in qualified data.

The final component of the qualification process is documentation of the NQA-1 requirements for specific data collection activities and documentation of the conformance of the actual data collection to those requirements. Within the VHTR program, these requirements are implemented through the VeryHigh-Temperature Gas-Cooled Reactor Technology Development Office Quality Assurance Program Plan (VHTR Technology Development Office [TDO] QAPP) (INL 2009b). The relevant documents that specify NQA-1 requirements and conformance for each data stream are given in the data stream sections of this report.

\section{FUEL FABRICATION DATA}

AGR-1 fuel is fabricated from low-enriched uranium oxycarbide kernels that are coated with multiple layers to form particles. The first coating is a low-density carbon layer (buffer layer), followed by a highdensity inner pyrolytic carbon (IPyC) layer, a silicon carbide (SiC) layer, and finally, a high-density outer pyrolytic carbon (OPyC) layer. Coating conditions for AGR-1 fuel were varied during particle fabrication to create a baseline and three variant particle composites. Process conditions for the baseline and three variants are described in Barnes (2006). Once the coatings are applied, thousands of particles are formed into cylindrical compacts using a graphite-resin matrix. Design specifications were established for this data stream, and the delivered hard copy data packages are the quality record that the design specifications were met or have been accepted as is.

\subsection{Description of the Data Stream}

The AGR-1 fuel fabrication data stream consists of properties obtained from measurements made on representative samples of fuel kernels, coated fuel particles, and fuel compacts. These properties are listed in the following sections along with specified acceptance criteria (Einerson 2006). The appropriate acceptance criterion depends on whether the property is a variable property or an attribute property. Variable properties are defined by a continuous distribution while attribute properties are discrete properties in the sense that the particle is either defective or not, in terms of that property. For variable properties, the criteria are stated in terms of a population mean and/or population dispersion with the mean having to lie within a specified interval. The acceptance criterion for attribute properties is stated in terms of the allowable fraction of defective particles. 


\subsubsection{Kernel Data}

A kernel composite consists of multiple kernel batches combined and mixed to ensure uniformity prior to sampling for acceptance. The composite lot of kernels (G73D-20-69302) used to make AGR-1 baseline and variant particles was fabricated by BWX Technologies (BWXT), now called Babcock and Wilcox, under a quality program that conformed to the requirements of NQA-1 1997 (ASME 1997) as per the requirements in effect at the time of kernel fabrication. Complete characterization data for this kernel lot are compiled in the Data Certification Package (BWXT 2005). Kernel composite properties included in NDMAS and corresponding specifications are listed in Table 1. Some kernel properties were also measured by ORNL prior to fabricating particles and compacts, but only the BWXT data were used for fuel certification. However, the Oak Ridge National Laboratory (ORNL) kernel data were included in NDMAS for comparison purposes.

Table 1. Properties and specifications for AGR-1 fuel kernels (Lot G73D-20-69302).

\begin{tabular}{|ll|}
\hline \multicolumn{1}{|c|}{ Kernel Property } & \multicolumn{1}{c|}{ Specified Range for Mean Value } \\
\hline U-235 enrichment (wt\%) & $19.80 \pm 0.10$ \\
Total Uranium (wt\%) & $\geq 87.0$ \\
Oxygen/uranium (atomic ratio) & $1.50 \pm 0.20$ \\
Carbon/uranium (atomic ratio) & $0.50 \pm 0.20$ \\
[Carbon+oxygen]/uranium (atomic ratio) & $\leq 2.0$ \\
Sulfur impurity (ppm $-\mathrm{wt})$ & $\leq 1,500$ \\
All other impurities ${ }^{(\text {a) }}$ & Various \\
Density (g/cm $\left.{ }^{3}\right)$ & $\geq 10.4$ \\
Diameter $(\mu \mathrm{m})$ & $350 \pm 10$ \\
Aspect ratio (sphericity or ellipticity) & Not specified \\
& \\
a. Not included in NDMAS because all impurities were below detection limits and within \\
$\quad$ specifications.
\end{tabular}

\subsubsection{Particle Data}

AGR-1 fuel kernels were shipped to ORNL where the coatings were added and the compacts fabricated under a quality program that conformed to the requirements of NQA-1 2000 (ASME 2000). Particles were coated in batches and particle composites were made from three or four coated batches. There are four particle composites for AGR-1 fuel-one for the baseline and each of the three variants. Complete characterization data for the four particles composites are compiled in data packages by Hunn and Lowden (2009, 2006a, 2006b, and 2006c). Particle properties included in NDMAS and corresponding specifications are listed in Table 2.

\subsubsection{Compact Data}

AGR-1 fuel compacts were fabricated by ORNL under a quality program that conformed to the requirements of NQA-1 2000 (ASME 2000). The same compacting process was used for the baseline fuel and all three variants, however the molding pressure did vary by compact. Complete characterization data for the four compact lots are compiled in data packages Hunn and Lowden (2009, 2006a, 2006b, and 2006c). Compact properties included in NDMAS and corresponding specifications are listed in Table 3. 
Table 2. Properties and specifications for AGR-1 coated particle composites.

\begin{tabular}{|ll|}
\hline \multicolumn{1}{|c}{ Property } & Specified Range for Mean Value \\
\hline Buffer density $\left(\mathrm{g} / \mathrm{cm}^{3}\right)$ & $0.95 \pm 0.15$ \\
Buffer thickness $(\mu \mathrm{m})$ & $100 \pm 15$ \\
IPyC density $\left(\mathrm{g} / \mathrm{cm}^{3}\right)$ & $1.90 \pm 0.05$ \\
IPyC thickness $(\mu \mathrm{m})$ & $40 \pm 4$ \\
IPyC anisotropy $(\mathrm{BAFo})$ & $\leq 1.035$ \\
SiC density $\left(\mathrm{g} / \mathrm{cm}^{3}\right)$ & $\geq 3.19$ \\
SiC thickness $\left(\mu \mathrm{m}^{3}\right)$ & $35 \pm 3$ \\
OPyC density $\left(\mathrm{g} / \mathrm{cm}^{3}\right)$ & $1.90 \pm 0.05$ \\
OPyC thickness $(\mu \mathrm{m})$ & $40 \pm 4$ \\
OPyC anisotropy $(\mathrm{BAF})$ & $\leq 1.035$ \\
Gold spot defect fraction & $5.0 \times 10^{-3}$ (Variants $\left.2 \& 3\right) ;$ \\
& $1.0 \times 10^{-3}($ Baseline and Variant 1$)$ \\
Defective SiC coating fraction & $\leq 1.0 \times 10^{-4}$ \\
Defective OPyC coating fraction & $\leq 3.0 \times 10^{-2}$ \\
IPyC anisotropy post compact deconsolidation (BAFo) & Not specified \\
OPyC anisotropy post compact deconsolidation (BAFo) & Not specified \\
Aspect ratio (sphericity) & Mean not specified ${ }^{(\mathrm{a})}$ \\
& \\
\hline a. Critical region is specified such that $\leq 1 \%$ of the particles shall have an aspect ratio $\geq 1.14$. \\
\hline
\end{tabular}

Table 3. Selected properties for AGR-1 compacts.

\begin{tabular}{|c|c|}
\hline Property & Specified Range for Mean Value \\
\hline Mean uranium loading (g U/compact) & $0.905 \pm 0.04$ \\
\hline $\mathrm{U}$ contamination fraction ${ }^{(\mathrm{a})}\left(\mathrm{g} \mathrm{U}_{\text {exposed }} / \mathrm{g} \mathrm{U}\right)$ & $\leq 1.0 \times 10^{-4}$ \\
\hline Iron content ( $\mu \mathrm{g} \mathrm{Fe}$ outside $\mathrm{SiC} /$ compact) & $\leq 25$ \\
\hline Chromium content ( $\mu \mathrm{g}$ Cr outside of $\mathrm{SiC} /$ compact) & $\leq 75$ \\
\hline Manganese content ( $\mu \mathrm{g}$ Mn outside of SiC/compact) & $\leq 75$ \\
\hline Cobalt content ( $\mu \mathrm{g}$ Co outside of $\mathrm{SiC} /$ compact) & $\leq 75$ \\
\hline Nickel content ( $\mu \mathrm{g}$ Ni outside of SiC/compact) & $\leq 75$ \\
\hline Calcium content ( $\mu \mathrm{g} \mathrm{Ca}$ outside of SiC/compact) & $\leq 90$ \\
\hline Aluminum content ( $\mu \mathrm{g} \mathrm{Al}$ outside of $\mathrm{SiC} /$ compact) & $\leq 45$ \\
\hline Titanium content ( $\mu \mathrm{g}$ Ti outside of SiC/compact) & Note $^{(b)}$ \\
\hline Vanadium content ( $\mu \mathrm{g} \mathrm{V}$ outside of $\mathrm{SiC} /$ compact) & Note $^{(b)}$ \\
\hline $\operatorname{Diameter}^{(\mathrm{c})}(\mathrm{mm})$ & $12.22-12.46$ \\
\hline Length $^{(\mathrm{c})}(\mathrm{mm})$ & $25.02-25.40$ \\
\hline Compact mass (g) & Not specified \\
\hline Molding pressure (MPa) & Not specified $^{(\mathrm{d})}$ \\
\hline \multirow{3}{*}{\multicolumn{2}{|c|}{$\begin{array}{l}\text { a. Value is an estimate of an attribute property, not the mean of a variable property. } \\
\text { b. Mean value specification of } \leq 400 \mu \mathrm{g} \text { Ti plus } \mathrm{V} \text { outside of } \mathrm{SiC} / \text { compact. } \\
\text { c. Allowable range corresponding to upper and lower critical limits specified with no compacts exceeding } \\
\text { the limits which requires } 100 \% \text { inspection of all compacts. }\end{array}$}} \\
\hline & \\
\hline & \\
\hline \multicolumn{2}{|l|}{ d. Not a variable, but a process condition that varied by compact. } \\
\hline
\end{tabular}




\subsubsection{Data Structure}

Prior to capturing the data in NDMAS, a hierarchal data structure based on components was created to make the data easier to process and analyze. A component is the generic name for the object or system being measured. In NDMAS, the fuel properties shown in Tables 1, 2, and 3 are known as response variables, and each response variable is a measurement or property associated with a component. For AGR-1 fuel, the component types are kernels, particles, particle layers, and compacts. Table 4 shows the component naming structure for each of the component types. There are 336 unique components for all four variants.

Table 4. Component types and names for AGR-1 fuel data.

\begin{tabular}{|c|c|}
\hline Component_type & Component_name \\
\hline Kernel Batch & LEU01 \\
\hline Buffer Layer ${ }^{\mathrm{a}}$ & LEU01-XXB \\
\hline IPyC Layer $^{\mathrm{a}}$ & LEU01-XXI and LEU01-XXT-ZI $^{\mathrm{b}}$ \\
\hline $\mathrm{SiC}_{\mathrm{Layer}}^{\mathrm{a}}$ & LEU01-XXS and LEU01-XXT-ZO ${ }^{\mathrm{b}}$ \\
\hline OPyC Layer ${ }^{\mathrm{a}}$ & LEU01-XXO \\
\hline Particle Composite & LEU01-XXT \\
\hline Compacts & LEU01-XXT-ZYY \\
\hline Compact Lot & LEU01-XXT-Z \\
\hline \multirow{2}{*}{\multicolumn{2}{|c|}{$\begin{array}{l}\text { a. Layers added to the kernels to make particles. } \\
\text { b. Some layer properties were measured on particles from deconsolidated } \\
\text { compacts. }\end{array}$}} \\
\hline & \\
\hline \multicolumn{2}{|c|}{$\begin{array}{l}\mathbf{X X}=\text { number associated with variant ( } 46=\text { Baseline, } 47=\text { Variant } 1,48=\text { Variant } 2 \text {, } \\
49=\text { Variant } 3 \text { ). }\end{array}$} \\
\hline \multicolumn{2}{|c|}{$\mathbf{Y Y}=$ compact number $(79$ each for Baseline, $\mathrm{V} 1$ and V3 variants, 67 for V2). } \\
\hline
\end{tabular}

Components in NDMAS are related to each other by an assembly tree. The component assembly tree for AGR-1 fuel data is shown in Figure 1. The compact lot at the base of the tree is composed of particles from the particle composite, which is composed of kernels from the kernel batch. Individual compacts are associated with (a branch of) the compact lot, and particle layers are associated with the particle composite. All the chemical properties in Table 3 are representative of the compact lot. The physical properties (diameter, length, and mass) are measured on individual compacts.

A careful review of the data packages identified the 33 unique response variables listed in Table 5. Where a mean value is indicated, there is also a response variable for the standard deviation and the number of samples in the population. There is no standard deviation for defect fractions because these are attribute properties (defective or not defective).

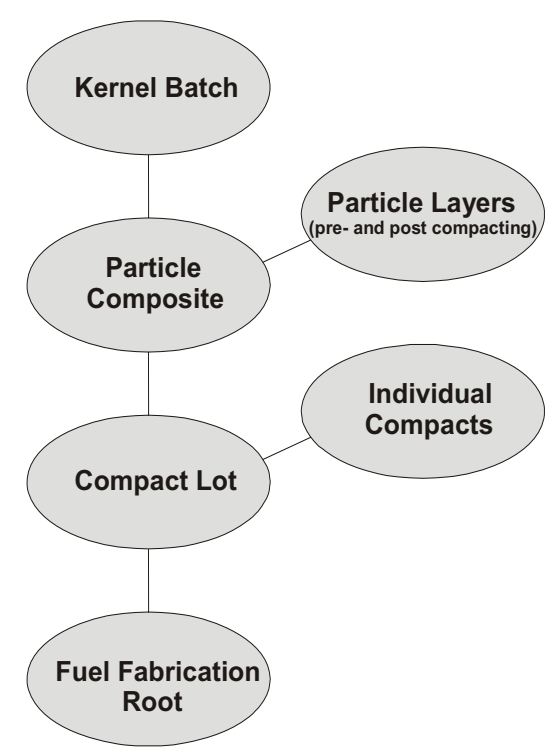

Figure 1. Fuel fabrication data component assembly tree structure. 


\subsection{Fuel Fabrication Data Processing within NDMAS}

The complete kernel data package (BWXT 2005) was transmitted to Idaho National Laboratory (INL) in hardcopy format. A summary of the kernel data package was transmitted in electronic Portable Document Format (PDF). The baseline and variant data packages for the particles and compacts (Hunn and Lowden 2009, 2006a, 2006b, 2006c) were transmitted in electronic PDF file format. None of the data in the data packages were in machine readable format. Before they could be captured into NDMAS, the data were entered into an Excel spreadsheet file named agr1_fuel_fab_data.xls.

The agr1_fuel_fab_data.xls spreadsheet file contains a separate worksheet for components, response variables, response variable values, and methods. Most of the response variable values were entered directly from the data packages into the worksheet. However, the mean and standard deviation density values for the buffer layers, IPyC layers and OPyC layers were not available for the entire population used to make the composite particles. Recall the composite particles were made from three or four coated batches. Means and standard deviation values for individual batches were reported in the data packages, but not for the entire population. In these cases, the batch mean and standard deviation values were used to calculate an overall mean and standard deviation (IPyC layers), or the density values of all samples in all the batches were used to calculate the mean and standard deviations for the entire population (OPyc layers). This was done in a separate Excel spreadsheet file called agr1_fuel_fab_density_calculations.xls and documented in Engineering Calculation and Analysis Report ECAR-824 (Sondrup 2009). The calculated density values were then entered into the agr1_fuel_fab_data.xls file. Both Excel files are archived on the NGNP SAS Server in the directory $\backslash$ Sasngnp \ngnp\NGNP_Data\Fuel_Fab $\backslash$ AGR $1 \backslash$.

Each worksheet of agr1 fuel fab data.xls was captured as a SAS dataset using the program \ISasngnp \ngnp \NDMAS Version 1.1.1 $\backslash$ Fuel_Fab $\backslash$ Capture_native_fuel_fab_data.egp. The four new SAS datasets (COMPONENTS, VARS, VALUES and METHODS) are stored in the SAS Library FUEL_FAB on the SAS Server. A different program, Capture_native_fuel_fab_data.egp, reads these SAS datasets and through a series of joins, filters, queries, and transposes, creates three additional datasets (EXP_COMP_for_upload, EXP_COMP_ATTRIB_for upload, EXP_ASSY_TREE_for_upload) to be appended to the appropriate files in the NDMAS Structured Query Language (SQL) database ("vault").

\subsection{Description of Fuel Fabrication Data Qualification}

Two general types of qualification tests are performed on data loaded into NDMAS:

- Capture tests, which verify that data captured and stored within NDMAS are identical to the source data provided to NDMAS.

- Accuracy tests, which verify the data are an accurate representation of the parameters they are intended to measure.

\subsubsection{Capture Tests}

The transmitted data are manually entered into the Excel spreadsheet file agr1_fuel_fab_data.xls. Once the data are transferred, every response variable value in the spreadsheet is manually checked against the values in the data packages to make sure they're identical. An independent person performs the comparison and the review is documented. The values used in the calculations for the calculated density values (see Section 3.2) are checked against the data in the data packages.

The second capture test is a referential integrity test to make sure that all components, component attributes and response variables, and response variable values are properly linked (see Section 3.1.4)

The third capture test verifies that the data in the SQL database are the same as the data loaded (pushed) into the SQL database. This test uses a SAS procedure (PROC-COMPARE) to compare the SAS dataset pushed to the SQL database with the database output. 
The final capture test is to compare the SQL database output with the original data in the data packages. This is another manual inspection similar to the first capture test. An independent person checks response variable values in the database against the data in the data packages and documents the results. Values determined from calculations (see Section 3.2) are compared against ECAR-824 (Sondrup 2009).

\subsubsection{Accuracy Tests}

The scope of accuracy testing is limited to the certification that AGR-1 fuel data for kernels, particles (including layers), and compacts meet specifications as outlined in Einerson (2006). Certification is performed by the data generators and documented in the subcontract deliverable data packages (BWXT 2005, Hunn and Lowden 2009, 2006a, 2006b, 2006c). Nonconformance reports are included in the data packages for any data that does not meet specifications. Certified data are verified and accepted by the contractor. Nonconformance data are reviewed and either rejected or accepted by the contractor.

The process of verifying that all data in the data packages meet specifications is a thorough process with multiple checks to ensure data accuracy. Because this process is so rigorous, no additional accuracy tests are planned for the fuel fabrication data.

\subsection{Verify Fuel Fabrication Data QA Documentation}

Kernels for AGR-1 fuel were produced under a quality program that conformed to the requirements of the 1997 version of NQA-1, which was in effect at the time of kernel fabrication. Quality requirements were passed to BWXT in Statement of Work SOW-427. Supplier audits were conducted to provide independent verification that quality requirements for the data were met.

Coated particles and compacts were produced under a quality program that conformed to the requirements of the NQA-1 2000 as implemented and documented by the fuel fabricator's QAPP (ORNL, 2006). Engineering Design File EDF-4380, "AGR-1 Fuel Product Specification and Characterization Guidance" (Barnes 2006) provides the requirements necessary for acceptance of the fuel manufactured for the AGR-1 irradiation test. Section 6.2 of EDF-4380 provides the property requirements for the heat treated compacts. EDF-4542, "Statistical Sampling Plan for AGR Fuel Materials" (Einerson 2006), provides additional guidance regarding statistical methods for product acceptance and recommended sample sizes. The procedures for characterizing and qualifying the compacts are outlined in ORNL product inspection plan AGR-CHAR-PIP-05. The inspection report forms generated by this product inspection plan document the product acceptance for the property requirements listed in Section 6.2 of EDF-4380 (Barnes 2006). Independent verification that quality requirements for the data were met is provided by supplier audits conducted by INL (Roberts and Barnes 2005a, 2005b).

\subsection{Fuel Fabrication Data Qualification Status}

A preliminary manual inspection was performed on a portion of the data in the Excel spreadsheet file agr1_fuel_fab_data.xls. The check found one error out of 230 values checked. The printed file was signed by the independent reviewer and placed in the project file. After capturing the data to the NDMAS SQL database, a manual inspection of data pulled from the vault was performed by an independent person who compared the data pulled from the vault to the data in the original data packages (BWXT 2005; Hunn and Lowden 2006a, 2006b, 2006c, 2009) and EDF-824 (Sondrup 2009). This inspection included verifying that data were associated with the correct component (Table 4), thus verifying referential integrity. Errors found in data in the vault were corrected. All fuel fabrication data were flagged as capture passed after the manual verification was complete and corrections were made.

As stated previously, no additional accuracy testing beyond what was done for data certification will be performed. Nevertheless, all data in the data packages met specifications with three exceptions. The first nonconformance is the $95 \%$ lower confidence limit for kernel uranium enrichment was $19.6962 \mathrm{wt} \%$, 
slightly less than the specification of $19.7 \mathrm{wt} \%$. The nonconformance report (included in the data package) shows the kernels were submitted for customer disposition and approved as is. The second nonconformance was also related to the kernels in that the carbon/uranium dispersion did not meet the specification as documented in Ebner (2005). Barnes (2005) provided the basis for the kernels to be used as is, and INL issued a Procurement Change Notice to BWXT releasing them from meeting the specification. The third nonconformance was that the length of some of the Baseline and Variant 2 compacts were slightly less than the specified minimum length. The nonconformance reports (provided as part of the data packages) document the recommendation and acceptance that the specimens be used as is for both irradiation and destructive characterization.

All of the fuel fabrication data entered into the NDMAS database has been verified against original documents provided by BWXT and ORNL. Independent verification that the data conform to requirements was obtained by site visits to BWXT and ORNL during data collection. All fuel fabrication data in the NDMAS data base is therefore qualified. A summary of the number of data records for each data package is shown in Table 6.

Table 6. Qualification status of fuel fabrication data.

\begin{tabular}{|l|c|c|}
\hline \multicolumn{1}{|c|}{ Data Package } & $\begin{array}{c}\text { Qualified } \\
\text { Records }\end{array}$ & $\begin{array}{c}\text { Total } \\
\text { Records }\end{array}$ \\
\hline BWXT Kernel Data for G73D-20-69300 & 27 & 27 \\
\hline Baseline Fabrication Data for LEU01-46T-Z & 466 & 466 \\
\hline Variant 1 Fabrication Data for LEU01-47T-Z & 457 & 457 \\
\hline Variant 2 Fabrication Data for LEU01-48T-Z & 409 & 409 \\
\hline Variant 3 Fabrication Data for LEU01-49T-Z & 460 & 460 \\
\hline Total & 1,819 & 1,819 \\
\hline
\end{tabular}

\subsection{Fuel Fabrication Data Problems and Resolution}

A problem with the AGR-1 fuel fabrication data is that it was not supplied in machine readable format. Some tables of data in the data packages appear to be scans of Excel spreadsheet files, but the Excel files were not readily available. The Excel files were scanned to preserve the signatures of operators, reviewers, and supervisors. These scanned images were high-quality reproductions and there were no problems discerning the data/numbers from the PDF files. Nevertheless, not having machine readable data requires each value to be entered by hand into a spreadsheet and checked manually. It is recommended that future fuel fabrication data packages be transmitted in machine readable format. Efforts should also be made, where possible, to standardize the formatting, especially for similar data types, with the goal of reducing any preprocessing required to handle distinct and unique files or tables. Having the data in standardized machine readable format may be more of a convenience issue than a problem, but it will be more efficient to process the data and reduce the possibility for errors. 


\section{FUEL IRRADIATION DATA}

\subsection{Description of the Data Stream}

The fuel irradiation experiment includes monitoring of the controlled gas flows to the capsule train that provide some temperature control and that collect emissions from the six fuel capsules and route them to the fission product monitoring system. Ten variables are measured as shown in Table 7 . These data are subsequently processed and stored in NDMAS. The data include flow rates of helium and neon gases to and from each capsule, gas pressure upstream of each capsule, moisture content of the gas flow mixture downstream of each capsule, and thermocouple temperatures at several locations within each capsule. Gas pressure, flow rates, and moisture content are also collected for the leadout system - the pressurized space around each capsule that prevents leakage of capsule gas flows into adjacent capsules. These data are generally collected at 5-minute intervals, except where data management issues during the first two reactor cycles (138B, 139A) provided only 2-hour interval data (INL Issue Communication and Resolution Environment [ICARE] NCR 42791 2008).

Table 7. Measurement variables in the fuel irradiation data stream.

\begin{tabular}{|lll|}
\hline Measurement Variable & \multicolumn{1}{c|}{ Description } & \multicolumn{1}{c|}{ Units } \\
\hline Moisture Content & Moisture content in gas flow line & Parts per million volume (ppmv) \\
Pressure & Pressure in gas flow line & Pounds per square inch atmosphere (psia) \\
Q_He & Helium flow rate & Standard cubic centimeters per min (sccm) \\
Q_Ne & Neon flow rate & sccm \\
Q_Total & Total outlet line gas flow rate & sccm \\
TC_1 & Thermocouple No. 1 temperature & ${ }^{\circ} \mathrm{C}$ \\
TC_2 & Thermocouple No. 2 temperature & ${ }^{\circ} \mathrm{C}$ \\
TC_3 & Thermocouple No. 3 temperature & ${ }^{\circ} \mathrm{C}$ \\
TC_4 & Thermocouple No. 4 temperature & ${ }^{\circ} \mathrm{C}$ \\
TC_5 & Thermocouple No.5 temperature & ${ }^{\circ} \mathrm{C}$ \\
\hline
\end{tabular}

The capsule thermocouples are of two different types and two different diameters, and thermocouple identification numbers may be associated with different positions and insertion depths in different capsules. A summary diagram of the thermocouples positions in the capsules is shown in Figure 2.

\subsection{Fuel Irradiation Data Processing within NDMAS}

Data processing and storage within NDMAS occurs via the following process. Raw data files covering about one week of measurements are placed in folders on the FSISC1 server as shown in Figure 3. Each folder contains one data file for each capsule with leadout system data included in the file for Capsule 1. A SAS Enterprise Guide project titled, "Update or build Irradiation dataset.egp," reads these data, assembles the data into a single SAS dataset, and stores the data in the NDMAS SQL database (vault). Processing and storage in NDMAS occurs approximately once per week so that several folders of data may be processed and entered as a single package. Data processing includes the following error checks to ensure that the data are accurately captured:

- Dates are checked for proper syntax and chronology.

- Data are checked for duplicated measurement times with conflicting variable values.

- Completed SAS datasets are visually inspected and compared against the raw data files. 


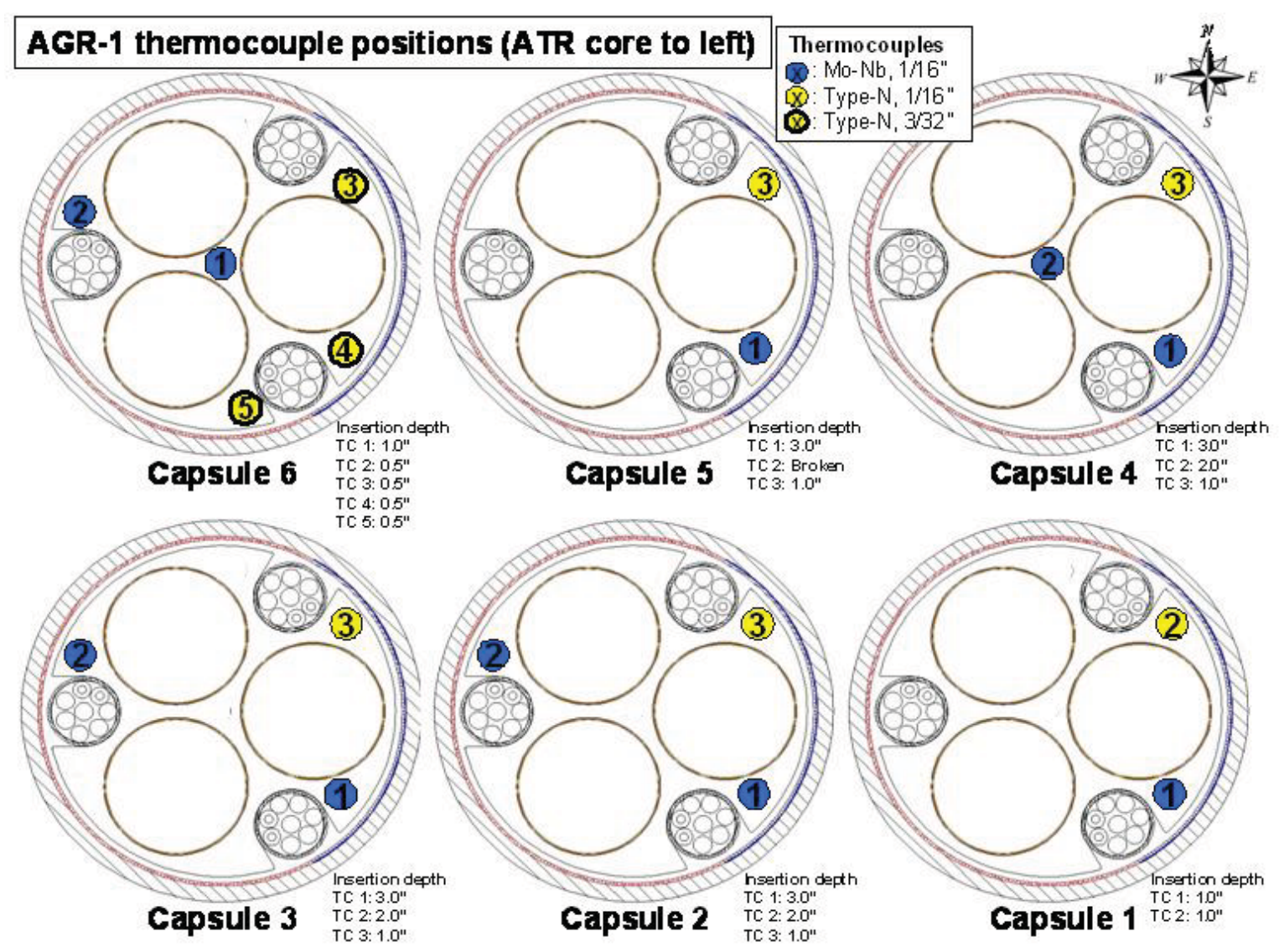

Figure 2. AGR-1 thermocouple descriptions and location information.

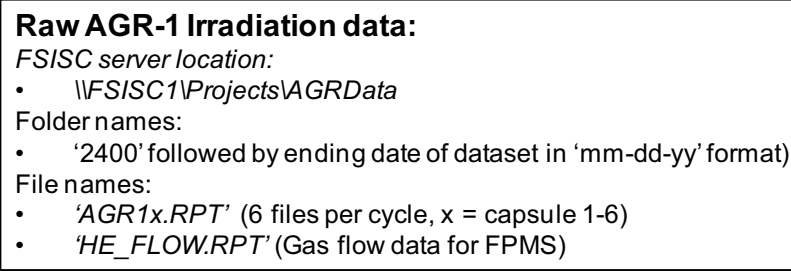

\begin{tabular}{|c|c|}
\hline \multicolumn{2}{|c|}{$\begin{array}{l}\text { AGR-1 Irradiation data processing steps: } \\
\text { - Copy new file folders from FSISC1 to } \\
\text { IISasngnpINGNPINGNP_DatalAGR-1| } \\
\text { - } \quad \text { FROM NDMAS directory, 'Vault_PushPull' }\end{array}$} \\
\hline 1) & $\begin{array}{l}\text { Run batch file 'update.bat' that creates/updates the list of relevant } \\
\text { folder names to be processed }\end{array}$ \\
\hline 2) & $\begin{array}{l}\text { Run Enterprise Guide Project file 'Update or build Irrad dataset.egp' to } \\
\text { construct SAS dataset for new data } \\
\text { - } \quad \text { Builds/appends all new data as SAS dataset } \\
\text { - } \quad \text { Pushes new data to SQL_NDMAS (NDMAS vault) } \\
\text { - } \quad \text { Checks that vault data is correctly entered }\end{array}$ \\
\hline 3) & Update log file 'Irradiation capture-push instructions and log.doc' \\
\hline 4) & $\begin{array}{l}\text { Run Enterprise Guide Project file 'Vault tests.egp' and examine any } \\
\text { failed data }\end{array}$ \\
\hline 5) & Resolve failed data as necessary \\
\hline 6) & $\begin{array}{l}\text { Run Enterprise Guide Project file 'Combine_Irrad_ATR_FPMS.egp' to } \\
\text { populate N_MART directory with new SAS data sets and cubes } \\
\text { containing updated irradiation data }\end{array}$ \\
\hline
\end{tabular}

Figure 3. Raw AGR-1 irradiation data and data processing steps. 
- A mountain standard date/time is assigned to each measurement that corrects for the switching between daylight-saving time (DST) and standard time that occurs in the raw data measurements. The switch to DST leads to repeated measurement times with different variable values, while the switch to standard time leads to a gap in measurement times.

The process of entering each data packages is recorded in an electronic log, with appropriate notes about any problems or corrections encountered. After being entered into the NDMAS SQL database, capture testing is performed for each data package to compare the database output with the SAS dataset from which it was built to ensure that the data were correctly stored. Results are stored in the electronic $\log$, "Irradiation capture-push instructions and log.doc."

\subsection{Description of Fuel Irradiation Data Qualification Tests}

Several tests, or analyses, are performed to attempt to identify data anomalies that may represent instrument drift or failure. Some of these methods are simple tests that, for example, check that the data are in a value range appropriate to the measurement. Others are more detailed analyses that rely on statistical analysis of past behavior as a guide to the range expected for new data values. These checks are programmed as a series of tests applied to each data package entered in the NDMAS SQL database. Anomalies identified by these tests are then examined with input from the technical leads and resolved to determine whether the anomalies represent (1) instrument failures or other errors that disqualify the data from use for their intended purpose, (2) values that are unusual but accurate, and therefore qualified data, or (3) instrument data that is reasonably precise, for use as trend data, but insufficiently accurate to be considered qualified data.

Range tests that detect data values outside expected ranges of measurement include the parameters and values listed in Table 8 .

Table 8. Range test values.

\begin{tabular}{ll}
\hline \multicolumn{1}{c}{ Parameter } & \multicolumn{1}{c}{ Requirement } \\
\hline Temperature & $0^{\circ} \mathrm{C}<\mathrm{X}<1,300^{\circ} \mathrm{C}$ \\
Gas pressure & $10 \mathrm{psia}<\mathrm{X}<20 \mathrm{psia}$ \\
Gas moisture content & $0 \mathrm{ppm}<\mathrm{X}<5 \mathrm{ppm}$ \\
Gas flow & $-2 \mathrm{sccm}<\mathrm{X}<52 \mathrm{sccm}$ \\
\hline
\end{tabular}

These range tests are based on a combination of physical limitations and/or requirements described in Technical and Functional Requirements (TFR) documents and other AGR-1 reference documents as follows:

- $\quad$ Requirements for gas moisture content are specified in TFR-248 (West et al. 2005), Section 3.1.2.4: "Moisture content of the inlet sweep gas shall be measured on the inlet side of the capsule at least once after each gas cylinder change and shall be $<5$ parts-per-million (ppm) $\mathrm{H}_{2} \mathrm{O}$."

- Based on the desired volume-averaged temperatures for the fuel in the experiment, the AGR-1 test train design requires temperature measurements in the $970-1,290^{\circ} \mathrm{C}$ range (Palmer 2006). As initial data from the experiment suggested that operating temperatures did not generally approach the higher end of that range, $1,300^{\circ} \mathrm{C}$ was selected as an upper limit for the NDMAS range test. The lower limit for temperatures should be limited by that of the water surrounding the capsule train, which enters the reactor vessel at an average temperature of $52^{\circ} \mathrm{C}$ and, at full power, exits the vessel at a temperature of $71^{\circ} \mathrm{C}$ (INL 2008). However, because the thermocouples commonly read low in the lowtemperature range, the prescribed lower limit for the range test is set at $0^{\circ} \mathrm{C}$.

- The range test for gas flow rates to the capsules is based on the nominal flow rates specified in TFR-248 (West et al. 2005), Section 3.1.2.2: "The tubing, valves, and MFCs shall be sized for a flow 
rate in each system up to 100 standard cubic centimeters per minute $(\mathrm{sccm})$ with a nominal flow rate of $50 \mathrm{sccm}$ thru the FPMS."

- Expert judgment. Monitoring data may be qualified according to physical principles or other information not revealed by statistical analyses. Temperature measurements, for example, are periodically compared to numerical simulations of heat flow that calculate temperatures at the thermocouple locations. Where the temperatures differ by greater than $\sim 50^{\circ} \mathrm{C}$, the thermocouples may be judged to have failed, sometimes by formation of a new junction. Such judgments, as recorded in controlled documents, are used to qualify the data where appropriate. For the AGR-1 experiment, examples include notes like the following from Maki (2007b):

These "TCs have provided measurements in excess of $200^{\circ} \mathrm{C}$ difference from calculated values (Ambrosek 2007). Such large differences indicate either the thermocouples have formed virtual junctions outside of the intended capsule or have developed another form of malfunction."

\subsection{Verify Fuel Irradiation Data QA Documentation}

The NGNP data collection process includes plans describing how data will be collected and the QA activities associated with those data. Review of those plans assures that the work will generate data of appropriate quality for use in the NQA-1 program. Metadata generated by the initial documentation, audits, and acceptance inspection provide the evidence that data meet the requirements of an NQA-1 data collection program. This is documented at the data stream level for fuel irradiation data. Documentation of the QA Program in NDMAS is primarily accomplished by references to documents. These include plans, audit reports, nonconformance reports, EDFs, and ECARs approved by the VHTR-TDO QA Lead.

The following documents provide evidence that the data for the fuel irradiation data stream meet the requirements of NQA-1, Part 1:

1. R. G. Ambrosek, "AGR-1 As-Run Thermal Evaluations - Cycle 138B, 139A and B," ECAR-102, Draft, December 4, 2007.

2. J. T. Maki, “AGR-1 Irradiation Test Specification,” EDF-4731, Rev. 1, July 2004.

3. J. T. Maki, AGR-1 Irradiation Experiment Test Plan, INL/EXT-05-00593, Rev. 2, March 2007.

4. J. T. Maki, 2007b, AGR-1 As-Run Analysis Status for FY-07, INL/EXT-07-13630, Rev 0, December 2007.

5. Email from John Maki to M. A. Plummer, with attachment, "AGR TC condition 10-28-08.doc"

6. A. J. Palmer, "Thermocouple recommendations for the AGR-1 test," EDF-6809, Rev. 0, May 2006.

7. P. B. West, G. A. Marts, E. W. Killian, J. K. Hartwell, and S. B. Grover, "Temperature Control and Off Gas Monitoring Systems for Advanced Gas Reactor Experiment AGR-1,” TFR-248, Rev. 1, March 2005.

8. P. B. West, G. A. Marts, E. W. Killian, J. K. Hartwell, and S. B. Grover, "Requirements for Design of the Advanced Gas Reactor Experiment AGR-1 for Irradiation in the Advanced Test Reactor," TFR-249, Rev. 1, December 2004.

9. INL ICARE NCR-42791, 2008, "ATR Experiment Data Collection System and Resulting Data," May 28, 2008. 


\subsection{Fuel Irradiation Data Analysis and Accuracy Testing}

In addition to the basic qualification tests described in Section 4.3, data from AGR-1 are being used to develop additional accuracy testing methods for the fuel irradiation data that can be used in AGR-2 and subsequent irradiation experiments. Focusing on detecting thermocouple failure, NDMAS has explored several different methods that can predict thermocouple temperatures from ATR/AGR operating parameters and thus provide an independent measure of thermocouple deviation from expected values. NDMAS is also examining data from thermocouples that were judged to have failed during AGR-1 but still appeared to provide realistic temperatures. Using those examples of failures and the data used to differentiate failed data, NDMAS anticipates that additional failure detection methods may be developed. Examples of tests being developed using analyses of AGR-1 data include:

- Independent temperature estimation based on historical temperatures and observed relationships between temperature and ATR operating conditions. For sufficiently short time periods, fission power heating should be well correlated with ATR configuration. Thermocouple temperatures should thus be well predicted from ATR configuration and the thermal conductivity of the gas flow mixture delivered to each capsule. Preliminary regression modeling demonstrates, for example, that temperatures in Capsule 6 are well predicted by a first-degree polynomial expression involving only the angle of control cylinder S1D2 and the neon fraction of gas flow to the capsule. Subsequent analysis will examine how the uncertainty of the predicted temperature varies with ATR configuration and length of time.

- Gas flow tests were used to identify several thermocouple failures in the first three AGR-1 cycles. NDMAS posits that periodic, automatic, preprogrammed gas-flow tests that sequentially alter gas flow mixtures in the capsules and automatically analyze temperature response to those changes should offer a robust means of identifying thermocouple deviations.

- While temperatures between capsules are well correlated, increased correlation between thermocouples in different capsules may reflect formation of a new junction in the thermocouple leads from the distal capsule. NDMAS is currently examining failed data from cases where this is believed to have occurred in order to develop tests that can identify those and other failures.

\subsection{Fuel Irradiation Data Qualification Status}

The overall qualification status for the separate AGR-1 fuel irradiation data packages is provided on the 'Qualification' page, INL NDMAS Web portal (https://sasweb.inl.gov). As of December 1, 2009, approximately 11 million irradiation data records have been stored in NDMAS, entered, and tested as 19 separate packages. The qualification status for these packages is summarized in Table 9.

\subsection{Fuel Irradiation Data Problems and Resolution}

\subsubsection{Data Collection Issues}

During most of the AGR-1 experiment, data from the capsule gas flow and temperature monitoring system were collected on one computer system and then transferred via floppy disk to another computer connected to the INL intranet. This system significantly limited NDMAS access to the data and thereby the frequency at which updates to NDMAS displays could be affected. In September 2009, a new data collection system was installed to record AGR and AGC data, and a temporary mode of file transfer was developed to provide data from that system to NDMAS. This altered the format of input files and NDMAS data capture systems were updated accordingly, yielding slight variants of the filenames and method described in Figure 3. During AGR-2 and subsequent experiments, it is expected that NDMAS will have a direct connection to the automated data collection system for the capsule gas flow and temperature monitoring data. 
Table 9. Qualification status of fuel irradiation monitoring data received as of August 1, 2009.

\begin{tabular}{|c|c|c|c|}
\hline & \multirow[b]{2}{*}{$\begin{array}{c}\text { Accuracy } \\
\text { Failed }\end{array}$} & \multirow[b]{2}{*}{ Qualified } & \multirow[b]{2}{*}{ Total } \\
\hline & & & \\
\hline Data Package & Records & Records & Records \\
\hline Irradiation data ending 20070703 & 113,439 & 546,478 & 659,917 \\
\hline Irradiation data ending 20090216 & $1,136,008$ & $6,742,058$ & $7,878,066$ \\
\hline Irradiation data ending 20090222 & 18,144 & 98,784 & 116,928 \\
\hline Irradiation data ending 20090301 & 18,144 & 98,784 & 116,928 \\
\hline Irradiation data ending 20090308 & 18,144 & 98,784 & 116,928 \\
\hline Irradiation data ending 20090315 & 18,144 & 98,784 & 116,928 \\
\hline Irradiation data ending 20090323 & 18,144 & 122,976 & 141,120 \\
\hline Irradiation data ending 20090412 & 54,324 & 368,196 & 422,520 \\
\hline Irradiation data ending 20090419 & 18,144 & 122,976 & 141,120 \\
\hline Irradiation data ending 20090621 & 39,332 & 242,908 & 282,240 \\
\hline Irradiation data from Folder 2400 04-26-09 & 18,144 & 122,976 & 141,120 \\
\hline Irradiation data from Folder $240005-24-09$ & 18,144 & 122,976 & 141,120 \\
\hline Irradiation data from Folders $240005-09-09$ and $240005-17-09$ & 35,883 & 243,207 & 279,090 \\
\hline Irradiation data from Folders $240005-31-09$ and $240006-07-09$ & 36,288 & 245,952 & 282,240 \\
\hline Irradiation data from Folders $240006-14-09$ and $240006-21-09$ & 16,128 & 266,112 & 282,240 \\
\hline Irradiation data from folders $06-28-09$ and $07-05-09$ & 39,332 & 114,684 & 154,016 \\
\hline Irradiation data from 2009 Sep 04 to 2009 Oct 12 & 69,623 & 47,658 & 117,281 \\
\hline Irradiation data from 2009-10-05 through 2009-11-02 & 27,162 & 79,568 & 106,730 \\
\hline Revised irradiation gas flow data from Sep 4 to Nov 92009 & 16 & 324 & 340 \\
\hline Totals & $1,712,687$ & $9,784,185$ & $11,496,872$ \\
\hline
\end{tabular}

Dates and times in the AGR-1 irradiation data collection system are recorded in USA Mountain Time, which shifts an hour ahead to DST in the spring, and back to standard time in the fall. This creates an apparent gap in the time domain data in the spring, and an hour of duplicate date-time records in the fall. These interruptions in the time domain sequences are generally identified automatically by the NDMAS data reading programs, and examination of those periods indicates that the data collection system transitioned to and from DST on dates inconsistent with the national implementation. Based on the records, the DST shifts appear to be made in a program that did not recognize the change in policy made in 2007. In addition, the Fall 2007 DST shift that should have been evident in the data was not found, and NDMAS assumed a change consistent with national policy. Dates and times where the AGR-1 DST shifts were made are shown in Table 10, with the corresponding correct dates for the shift and shift dates not accounting for the DST change made in 2007 . To provide a consistent time and record for all data sets, it is recommended that subsequent AGR experiments also maintain a Mountain Standard Time (MST), or other standard time, date/time stamp for all records. 
Table 10. Daylight saving time changes recorded in AGR-1 records, with corresponding dates and times for the correct time of change and time using pre-2007 policy.

\begin{tabular}{|c|c|c|c|}
\hline & \multicolumn{3}{|c|}{ DST Shift Date/Time } \\
\hline & AGR-1 & U.S. & Pre-2007 U.S. \\
\hline Fall 2006 & Insufficient data & 10/29/06 2:00 AM & 10/29/06 2:00 AM \\
\hline Spring 2007 & 04/01/07 2:00 AM & 03/11/07 2:00 AM & 04/01/07 2:00 AM \\
\hline Fall 2007 & Not found & 11/04/07 2:00 AM & 10/28/07 2:00 AM \\
\hline Spring 2008 & Insufficient data & 03/09/08 2:00 AM & 04/06/08 2:00 AM \\
\hline Fall 2008 & 10/26/08 3:00 AM & 11/02/08 2:00 AM & 10/26/08 2:00 AM \\
\hline Spring 2009 & 04/05/09 2:00 AM & 03/08/09 2:00 AM & 04/05/09 2:00 AM \\
\hline Fall 2009 & 11/01/09 2:00 AM & 11/01/09 2:00 AM & 2:00 AM \\
\hline
\end{tabular}

\subsubsection{Failed Irradiation Data}

Data problems with the irradiation experiment include failures in the data collection system and failures in accuracy. The former failure occurred during all of the first AGR-1 cycle (ATR Cycle 138B) and the first week of the second AGR-1 cycle (ATR Cycle 139A) when all of the electronic data files were irretrievably lost. Available data from that period are therefore restricted to that contained in hardcopy log sheets which contain control thermocouple data and sweep gas data recorded at 2-hour intervals. Because data collection for that period did not conform to TFR-248 (West et al. 2005) requirements for the experiment, INL ICARE NCR 42791 (2008) was issued to address the issues associated with that data loss and ensure that future data management would comply with TFR-248 (West et al. 2005).

Data from the fuel irradiation data monitoring stream that have failed accuracy tests are plotted in Figures $4,5,6$, and 7 . The failed data generally reflect shakedown issues that occurred during the first three cycles, except for minor anomalies that likely reflect short-term interruptions to recording systems. A summary of the explanations for the failed data precedes each figure.

\subsubsection{Gas moisture Content Data}

Most of the failed gas moisture data (see Figure 4) reflects "repair of minor gas leaks, repair of faulty gas line valves; the need to position moisture monitors in regions of low radiation fields for proper functioning; the enforcement of proper online data and the need to monitor thermocouple performance." (Maki 2007b). By the end of the third cycle, it was concluded that the moisture monitors had degraded and then failed because of radiation damage to the sensor electronics. This implied that all moisture readings through the first three cycles were erroneous. During the reactor outage, between the third and fourth cycles, the moisture monitors were replaced with new sensors and relocated to an area with significantly reduced radiation fields. Other anomalous data were identified in Capsules 5 and 6 on one occasion in March 2008 and a second occasion in January 2009. 


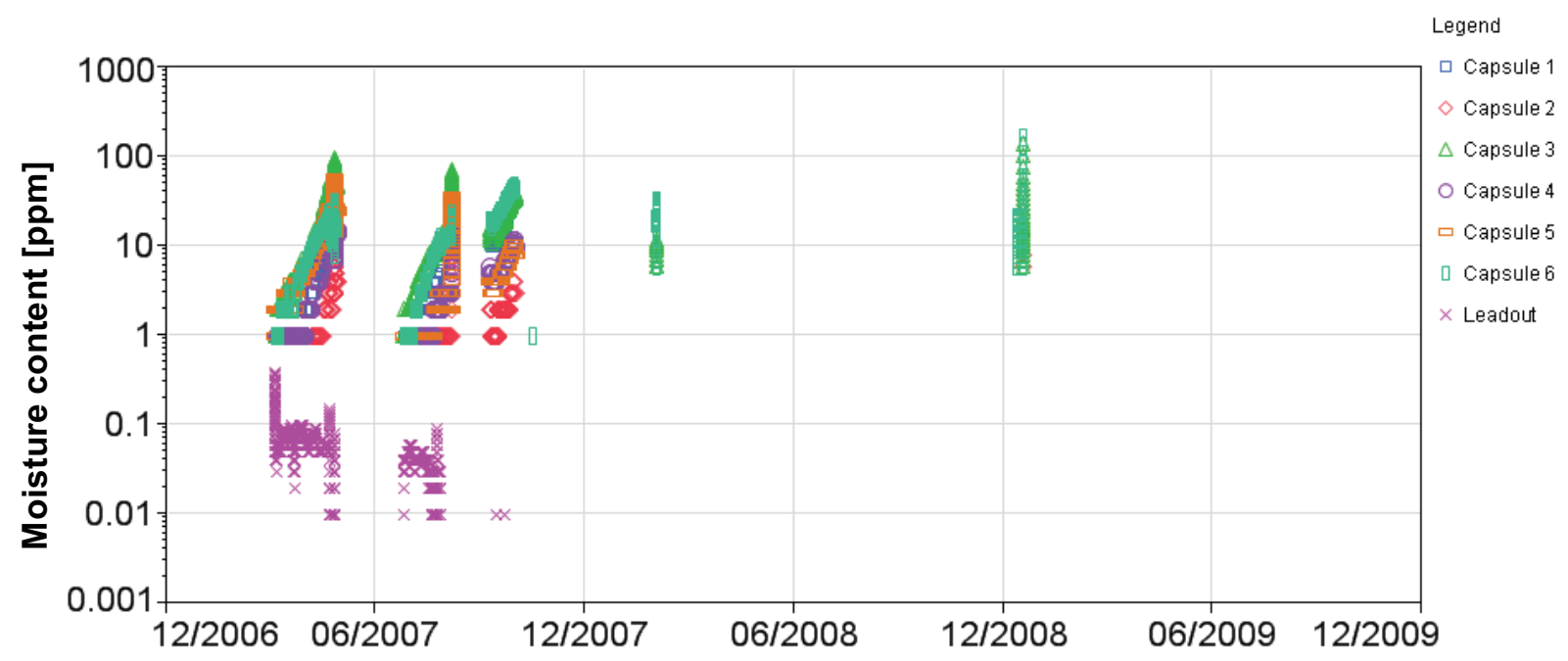

Figure 4. Gas moisture content data (log scale) from AGR-1 that failed accuracy tests implemented by NDMAS.

\subsubsection{Gas Pressure Data}

Relatively few anomalous gas pressure events (see Figure 5) were identified. The first of these was likely associated with the replacement of gas moisture monitoring instruments, as described above. Similarly, anomalies in gas moisture data in March 2007 and January 2009 correspond to anomalies in gas moisture content data and may thus reflect maintenance activities.

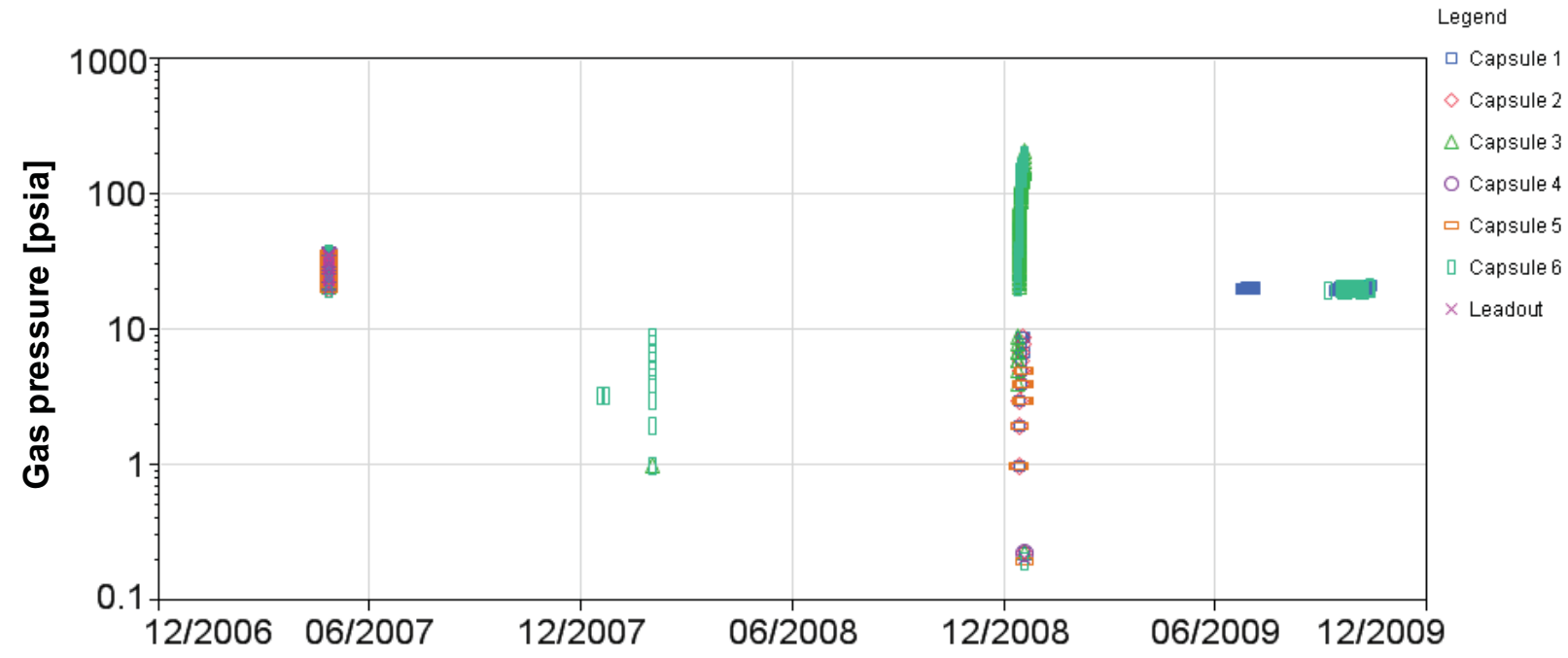

Figure 5. Gas pressure data (log scale) from AGR-1 that failed accuracy tests implemented by NDMAS.

\subsubsection{Gas Flow Data}

Relatively few anomalous gas flow events were identified (see Figure 6), and with the exception of erroneous values from September 14, 2009, these anomalies reflected only slightly excessive flows in the range of 52 to $63 \mathrm{sccm}$. Out of range values in September 2009 were associated with a data gap in flow values for many of the capsules and are believed to reflect an adjustment to the data collection system. 


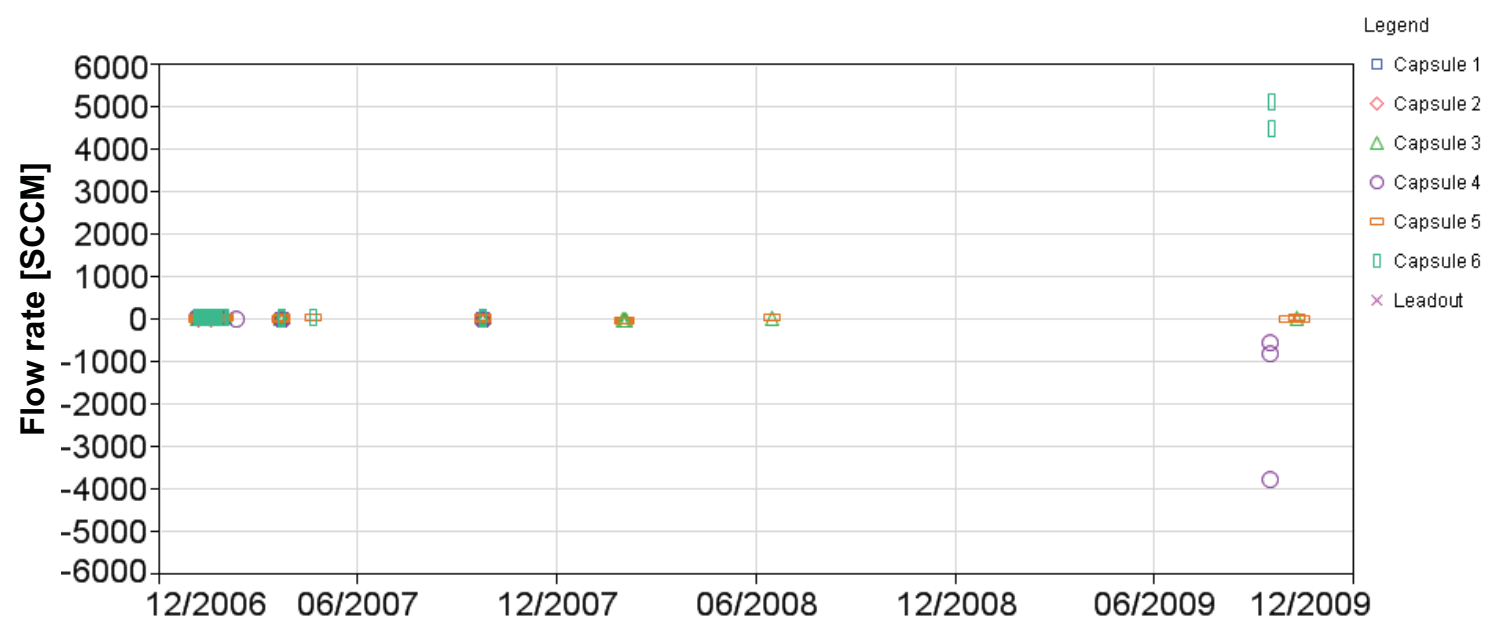

Figure 6. Gas flow data from AGR-1 that failed accuracy tests implemented by NDMAS.

\subsubsection{Thermocouple Data}

Thermocouple failures that occurred during fabrication and the experiment were generally identified by the NGNP technical leads via gas flow tests or the comparison of neutron transport and heat flow within the capsules to numerical simulations. The failure chronology, as provided to NDMAS (personal communication, J. Maki to M. Plummer, Oct 28, 2008; Maki 2007b), is summarized in Table 11 and the failed data, as recorded in NDMAS, are plotted in Figure 7.

Table 11. Chronology of thermocouple failures during AGR-1, based on NDMAS-assigned failure dates. Information provided to NDMAS is shown in the "AGR TC Table" column. Because thermocouple failure does not always coincide with complete failure of the instrument to respond to reactor conditions, the date of that failure, if it occurred, is shown as the 'Failure to respond' date.

\begin{tabular}{|c|c|c|c|}
\hline $\begin{array}{l}\text { NDMAS-Assigned } \\
\text { Failure Date }\end{array}$ & Thermocouple & AGR thermocouple Table Notes & $\begin{array}{l}\text { 'Failure to respond' } \\
\text { Date }\end{array}$ \\
\hline $11 / 30 / 06$ & Capsule 5 TC-2 & Failed $11 / 30 / 06$ during fabrication & $11 / 30 / 06$ \\
\hline $11 / 30 / 06$ & Capsule 1 TC-1\# & Failed $11 / 30 / 06$ during fabrication & $12 / 02 / 07$ \\
\hline $11 / 30 / 06$ & Capsule 2 TC- $1 *$ & Failed 11/30/06 during fabrication & 04/28/08 \\
\hline 04/01/07 & Capsule 3 TC-2* & Failed mid $2^{\text {nd }}$ cycle & $04 / 29 / 08$ \\
\hline $04 / 22 / 07$ & Capsule 2 TC- $2 \dagger^{*}$ & Failed $04 / 22 / 07$ after end of $2^{\text {nd }}$ cycle & $04 / 28 / 08$ \\
\hline $9 / 22 / 07$ & Capsule 1 TC-2† & Failed mid $3^{\text {rd }}$ cycle & $9 / 22 / 07$ \\
\hline $09 / 30 / 07$ & Capsule 2 TC-3 & Failed $09 / 30 / 07$ after end of $3^{\text {rd }}$ cycle & $04 / 28 / 08$ \\
\hline $12 / 02 / 07$ & Capsule 3 TC-3 & Failed $12 / 02 / 07$ after end of $4^{\text {th }}$ cycle & $11 / 30 / 07$ \\
\hline $03 / 09 / 08$ & Capsule 3 TC-1 & Failed $03 / 09 / 08$ after end of $6^{\text {th }}$ cycle & NA \\
\hline $10 / 16 / 08$ & Capsule 4 TC-3 & Failed $10 / 16 / 08$ after end of 9 th cycle & $11 / 20 / 08$ \\
\hline \multicolumn{4}{|c|}{$\begin{array}{l}\text { \# As a result of the neon injection tests, it was concluded that Capsule } 1 \text { TC-1 had failed via formation of a virtual junction } \\
\text { near the location of Capsule } 6 \text {. }\end{array}$} \\
\hline \multicolumn{4}{|c|}{ Capsule 2 TC-2 shows declining temp while other thermocouples ( 1 and 3 ) are increasing, beginning early in the 2 nd cycle. } \\
\hline \multicolumn{4}{|c|}{$\$ \quad$ No apparent failure before total failure in second full-power period of 3rd cycle (139B). } \\
\hline \multicolumn{4}{|c|}{$\begin{array}{l}\text { * These thermocouples provided measurements in excess of } 200^{\circ} \mathrm{C} \text { difference from calculated values (Ambrosek 2007), } \\
\text { indicating that the thermocouples had either formed virtual junctions outside of the intended capsule or had developed } \\
\text { another form of malfunction. }\end{array}$} \\
\hline
\end{tabular}



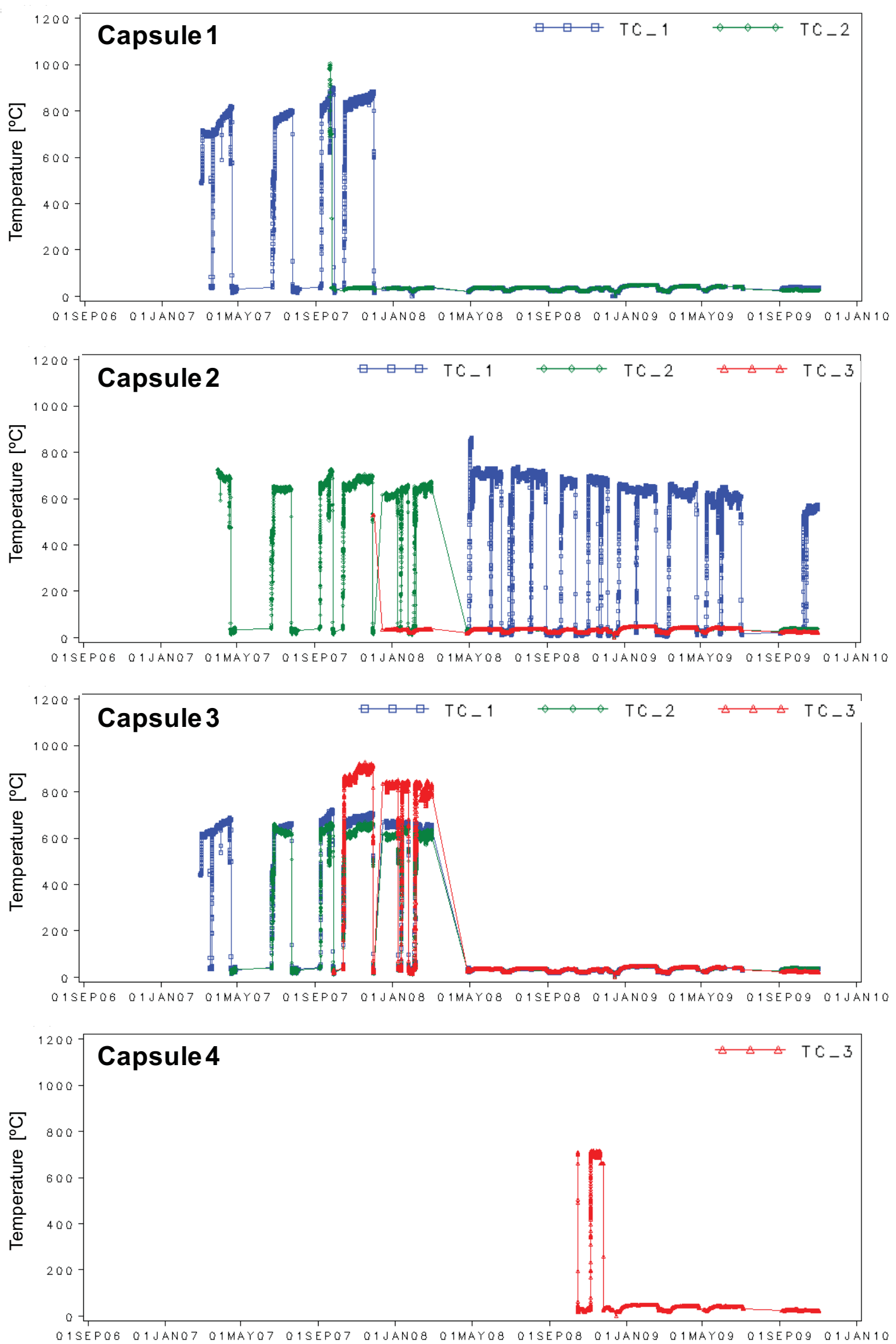

Figure 7. Failed thermocouple temperature data from AGR-1, based on information provided to NDMAS by the NGNP technical leads. 


\section{FPMS DATA}

\subsection{Description of the Data Stream}

The gas effluent in each of the six capsules of the AGR-1 experiment is monitored by a radiation detector system consisting of a sodium iodide detector for measuring gross gamma activity and a high purity germanium spectrometer for quantifying isotopic activities. The collection of radiation measurement systems is known as the AGR-1 FPMS. The two types of FPMS data provided to NDMAS by the FPMS technical staff are:

- Processed (by FPMS staff) spectral files consisting of radionuclide release rates (in atoms/sec) and release-to-birthrate (R/B) ratios for 12 noble gas fission product gases $(\mathrm{Kr}-85 \mathrm{~m}, \mathrm{Kr}-87, \mathrm{Kr}-88, \mathrm{Kr}-89$, $\mathrm{Kr}-90, \mathrm{Xe}-131 \mathrm{~m}, \mathrm{Xe}-133, \mathrm{Xe}-135, \mathrm{Xe}-135 \mathrm{~m}, \mathrm{Xe}-137, \mathrm{Xe}-138$, and Xe-139). These data are classified as Qualified data.

- Gross gamma data consisting of sequential 8-hour binary files of 3.5-sec gross gamma counts.

Gross gamma count data are processed by NDMAS to provide graphical displays on the INL NDMAS Web portal (https://sasweb.inl.gov). These data are used to provide an early indicator of potential fuel particle failures. As such, they do not undergo any capture or accuracy testing within NDMAS, are not qualified, and are not stored in the NDMAS SQL database. The native binary files are

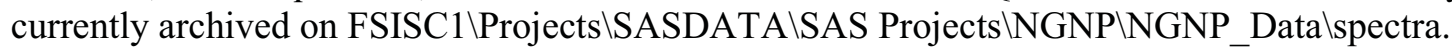

Separate spectral release rate and R/B ASCII text files for each capsule were supplied to NDMAS at the end of each reactor cycle (six release files and six R/B files) throughout the AGR-1 experiment. In February 2010, FPMS data from all of 13 cycles of the AGR-1 test were reprocessed to correct the Kr-87 activity for interference from $\mathrm{Xe}-138$ and the $\mathrm{Kr}-88$ activity for spectral shifts as documented in PLN-3392, "Fission Product Monitoring System PCGAP Software Modifications and Operability Test Plan" (in press).

Each data record in the processed FPMS files consists of fission product release rates (in atoms/sec) or R/B data (unitless) with associated uncertainty (\%) over nominal 8-hour counting intervals as shown in Table 12. A detailed description of the FPMS data processing done by the FPMS technical staff can be found in the User's Guide for the Fission Product Monitoring System (Drigert, Scates, and Walter 2009) and PLN-3392. Qualification of the processed data in these files is performed outside of NDMAS by the FPMS technical staff and documented in ECAR-907 (Scates 2010) for all reactor cycles. This ECAR is approved by the VHTR-TDO QA Lead and the VHTR-TDO Irradiations Technical Lead. Data capture testing was performed within NDMAS to ensure the data stored in the database are identical to the original data (see next section). In addition, NDMAS performed data analysis (e.g., statistical distributions and plotting) and is displaying the results on the NDMAS Web portal to assist researchers in data interpretation.

\subsection{FPMS Data Processing within NDMAS}

\subsubsection{Gross Gamma Counts}

The gross gamma count data are captured in their original binary file format, converted to text data, processed within SAS, and displayed as graphs on the "FPMS Gross Gamma Data" page of the NDMAS Web Portal (https://sasweb.inl.gov). The raw binary (.dgt) files are automatically transferred on a daily

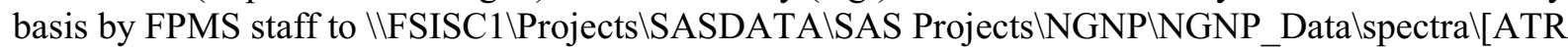
cycle\#] where they are archived and accessed for SAS plotting.

Table 12. FPMS release and R/B data provided to NDMAS.

Variable Name

Description 


\begin{tabular}{|c|c|}
\hline Variable Name & Description \\
\hline SpecID & $\begin{array}{l}\text { Sample record name in the form } \mathrm{GxYmmddhhzz}, \text { where } \mathrm{Gx}=\text { detector number } \\
(\mathrm{G} 1-\mathrm{G} 7), \mathrm{Y}=\text { last digit of year, } \mathrm{mm}=\text { month }(01-12), \mathrm{dd}=\text { day of month }(01 \\
-31), \mathrm{hh}=\text { hour of day }(00-23), \mathrm{zz}=\text { index number for detector restart }\end{array}$ \\
\hline Date/Time & Local (MST or MDT) start time of sample \\
\hline$<$ flow $>$ & Detector gas flow rate in standard $\mathrm{cm}^{3}$ per second \\
\hline $\mathrm{n}$ & Number of gas flow readings used in the FPMS post-processing \\
\hline $\mathrm{Kr} \_85 \mathrm{M} \_\mathrm{Rel} /[\mathrm{Err}$ & Release rate (atoms/s) and associated error $(\%)$ for $\mathrm{Kr}-85 \mathrm{~m}$ (2 variables) \\
\hline $\mathrm{Kr} \_87$ Rel/_Err & Release rate (atoms/s) and associated error (\%) for $\mathrm{Kr}-87$ (2 variables) \\
\hline $\mathrm{Kr} \_88 \_\mathrm{Rel} /$ Err & Release rate (atoms/s) and associated error (\%) for $\mathrm{Kr}-88$ (2 variables) \\
\hline Kr_89_Rel/_Err & Release rate (atoms/s) and associated error (\%) for $\mathrm{Kr}-89$ (2 variables) \\
\hline Kr_90_Rel/_Err & Release rate (atoms/s) and associated error (\%) for $\mathrm{Kr}-90$ (2 variables) \\
\hline Xe_131M_Rel/_Err & Release rate (atoms/s) and associated error (\%) for Xe- $131 \mathrm{~m}$ ( 2 variables) \\
\hline Xe_133_Rel/_Err & Release rate (atoms/s) and associated error (\%) for Xe-133 (2 variables) \\
\hline Xe_135_Rel/_Err & Release rate (atoms/s) and associated error (\%) for Xe-135 (2 variables) \\
\hline Xe_135M_Rel/_Err & Release rate (atoms/s) and associated error (\%) for Xe-135m (2 variables) \\
\hline $\mathrm{Xe}$-137_Rel/_Err & Release rate (atoms/s) and associated error (\%) for Xe-137 (2 variables) \\
\hline Xe_138_Rel/_Err & Release rate (atoms/s) and associated error (\%) for Xe-138 (2 variables) \\
\hline Xe_139_Rel/_Err & Release rate (atoms/s) and associated error (\%) for Xe-139 (2 variables) \\
\hline $\mathrm{Kr} \_85 \mathrm{M} \_\mathrm{Rat} / \mathrm{REr}$ & $\mathrm{R} / \mathrm{B}$ ratio and associated error $(\%)$ for $\mathrm{Kr}-85 \mathrm{~m}$ \\
\hline $\mathrm{Kr} \_87 \_\mathrm{Rat} / \mathrm{REr}$ & $\mathrm{R} / \mathrm{B}$ ratio and associated error $(\%)$ for $\mathrm{Kr}-87)$ \\
\hline Kr_88_Rat/_REr & $\mathrm{R} / \mathrm{B}$ ratio and associated error $(\%)$ for $\mathrm{Kr}-88$ \\
\hline Kr_89_Rat/_REr & $\mathrm{R} / \mathrm{B}$ ratio and associated error $(\%)$ for $\mathrm{Kr}-89$ \\
\hline Kr_90_Rat/REr & $\mathrm{R} / \mathrm{B}$ ratio and associated error $(\%)$ for $\mathrm{Kr}-90$ \\
\hline Xe_131M_Rat/_REr & $\mathrm{R} / \mathrm{B}$ ratio and associated error $(\%)$ for $\mathrm{Xe}-131 \mathrm{~m}$ \\
\hline Xe_133_Rat/_REr & $\mathrm{R} / \mathrm{B}$ ratio and associated error $(\%)$ for $\mathrm{Xe}-133$ \\
\hline Xe_135_Rat/_REr & $\mathrm{R} / \mathrm{B}$ ratio and associated error $(\%)$ for $\mathrm{Xe}-135$ \\
\hline Xe_135M_Rat/_REr & $\mathrm{R} / \mathrm{B}$ ratio and associated error $(\%)$ for $\mathrm{Xe}-135 \mathrm{~m}$ \\
\hline Xe_137_Rat/_REr & $\mathrm{R} / \mathrm{B}$ ratio and associated error $(\%)$ for $\mathrm{Xe}-137$ \\
\hline Xe_138_Rat/_REr & $\mathrm{R} / \mathrm{B}$ ratio and associated error $(\%)$ for $\mathrm{Xe}-138$ \\
\hline Xe_139_Rat/_REr & $\mathrm{R} / \mathrm{B}$ ratio and associated error $(\%)$ for $\mathrm{Xe}-139$ \\
\hline
\end{tabular}

Specific data processing steps for the SAS plotting of the gross gamma data are:

1. Daily run of 'Read_Binary_GrossGamma.bat,' located on \ISasngnp\NGNP\NDMAS Version 1.1\FPMS \GrossGamma\Capture.

2. The above batch file runs the SAS 9.1 code, 'Read_Binary_GrossGamma.sas.' This code searches the above source (.dgt) file folder any new files that have not been captured since the previous search, invokes a Fortran executable code to convert these binary files to text, and appends the count data into seven detector SAS data sets located on $\backslash$ lsasngnp $\backslash$ Ngnp $\backslash$ Data\FPMS (e.g., dtr1_grgamma.sas7bdat). The SAS metadata library for these files is FPMS.

3. Plots of the data are developed in the SAS E-Guide file, 'GrossGammaPlots.egp,' located on IISasngnp $\backslash$ NGNP $\backslash$ NDMAS Version 1.1\FPMS $\backslash$ GrossGamma $\backslash$ Graphics. This file contains three stored 
processes that control processing of the (1) "Plot Recent Gross Gamma Counts" - a 7-day plot of the most recent processed data, (2) "Plot Short Term Gross Gamma Counts" - a plot of the data for any user-selected time period $<24$ hours, and (3) "Plot Long Term Gross Gamma Counts" - a plot of the data for any user-selected time period $\geq 24$ hours.

\subsubsection{Release and R/B Data}

The six capsule-specific release rate and R/B data files for each ATR reactor cycle are pushed by

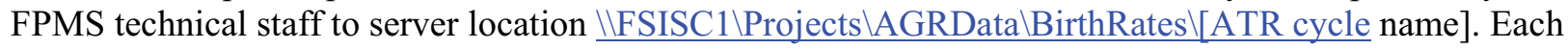
cycle-specific set of files comprised an FPMS data package for processing within NDMAS. NDMAS processing for these files is summarized in Figure 8.

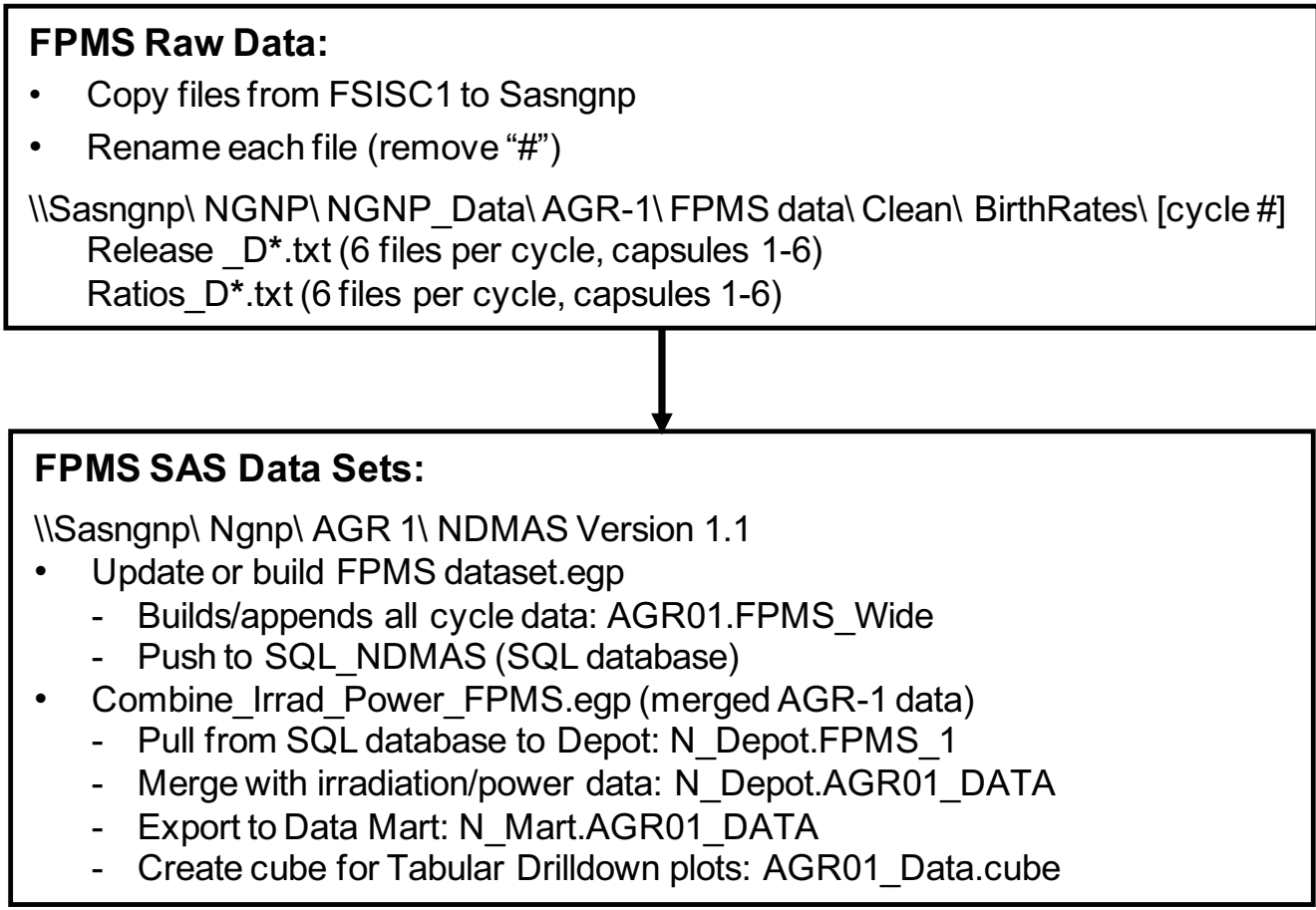

Figure 8. SAS processing of FPMS data (radionuclide release rates and R/B ratios).

\subsection{Description of FPMS Data Qualification Tests}

The FPMS release and R/B data are in the Qualified data category. Two general types of qualification tests are performed on these data in NDMAS:

- Capture tests verify that there are no simple raw data processing errors in the source files and that the data captured and stored within the NDMAS database are identical to the source data provided to NDMAS.

- Accuracy tests verify that the data are an accurate representation of the system parameters they are intended to measure.

The qualification tests for the FPMS release and R/B data are listed in the 'NDMAS Test Library.srx'on 'Qualification' page of the NDMAS Web portal (https://sasweb.inl.gov). Two time-related capture tests are currently performed on these data using the SAS E-Guide file, ' $\backslash$ SSASNGNP $\backslash$ NGNP $\backslash N D M A S$ Version 1.1 \Vault_PushPull\Update or build FPMS dataset.egp' to check that there are no obvious data processing errors in the source files: 
- Test to ensure data records are in chronological order; if a data record is not in chronological order, an error is recorded for that record in the SAS dataset.

- Test to ensure the date/time entries for each data record are consistent with those in the SpecID variable in the data file (sample record ID). SpecID is in the form "GxYmmddhhzz," where $\mathrm{Gx}=$ detector number (G1-G7), $\mathrm{y}=$ last digit of year (e.g., 9 for 2009), $\mathrm{mm}=$ month $(00-12)$, $\mathrm{dd}=$ day of month (01-31), hh = hour of day (00-23), and $\mathrm{zz}=$ index number for detector restart (e.g., $00=$ initial start). If the SpecID does not match the sample date/time, an error is recorded for that record in the SAS dataset.

A final capture test is performed in the same SAS E-Guide file to verify that the data captured and stored within the NDMAS SQL database are identical to the source data provided to NDMAS. This test uses a SAS procedure (PROC-COMPARE) to compare the SAS dataset used as input to the SQL database with the SQL database output. Records of these test results are maintained for each FPMS reactor cycle data package processed within NDMAS.

The accuracy of the release and R/B data files for all 13 AGR-1 reactor cycles is being verified by the FPMS technical staff in ECAR-907. After ECAR approval notification, the NDMAS staff will log an ECAR "accuracy test" as complete in the SQL database for each AGR-1 cycle data package. FPMS release and R/B data for a given reactor cycle are considered Qualified after successful completion and documentation (i.e., database logging) of both the NDMAS capture tests and approval of the ECAR.

\subsection{Verify FPMS Data QA Documentation}

The data collection process includes writing a plan describing how data will be collected and the QA activities associated with that data. Review of the plan assures that the planned work will generate data of appropriate quality for use in the NQA-1 program. Metadata generated by the initial documentation, audits, and acceptance inspection provide the evidence that data meet the requirements of an NQA-1 data collection program. This is documented at the data stream level and, for FPMS data, at the data package level (for each end-of-cycle file package).

Documentation of the QA Program in NDMAS is primarily accomplished by reference to documents. These include plans, audit reports, nonconformance reports, and ECARs approved by the VHTR-TDO QA Lead. The following documents provide evidence that the data for the FPMS data stream meet the requirements of NQA-1, Part 1:

1. J. T. Maki, AGR-1 Irradiation Experiment Test Plan, INL/EXT-05-00593, Rev 2, March 2007.

2. D. M. Scates and J. K. Hartwell, Fission Product Monitoring System Operability Test Plan for the AGR-1 Experiment: Phase II, PLN-2350, December 2006.

3. D. M. Scates, "Fission Product Monitoring System PCGAP Software Modification and Operability Test Plan," PLN-3392, in press February 2010.

4. M. W. Drigert, D. M. Scates, and J. B. Walter, "Users' Guide for the Fission Product Monitoring System," GDE-503, Rev 0, 04/16/09.

5. J. K. Hartwell, J. B. Walter, D. M. Scates, and M. W. Drigert, Determination of the AGR-1 Capsule to FPMS Spectrometer Transport Volumes from Leadout Flow Test Data, INL/EXT-07-12494, May 2007.

6. D. M. Scates, "Release to Birth Ratios for AGR-1 Operating Cycles 138B through 145A," ECAR-907 (in press February 2010). 


\subsection{FPMS Data Analysis and Web Display}

Data analyses and presentations not currently associated with FPMS data qualification are performed on the FPMS data stored in the NDMAS SQL database to assist in data interpretation by researchers. The results are displayed on the NDMAS Web portal (https://sasweb.inl.gov, 'FPMS Release and R/B Data' page). ATR cycle-specific release and R/B datasets are accessible using a SAS "Data Exploration" tool which allows user selection of display variables; filtering by date, capsule, or response value; plotting of small datasets; and download of the data to an Excel spreadsheet. Comprehensive data plots and distribution analyses for all cycles and capsules are also posted on the web page (Figures 9 through 12). Additional FPMS data analyses (e.g., correlation of fission product release rates with capsule thermocouple temperatures) can be added to the Web page at the request of users.

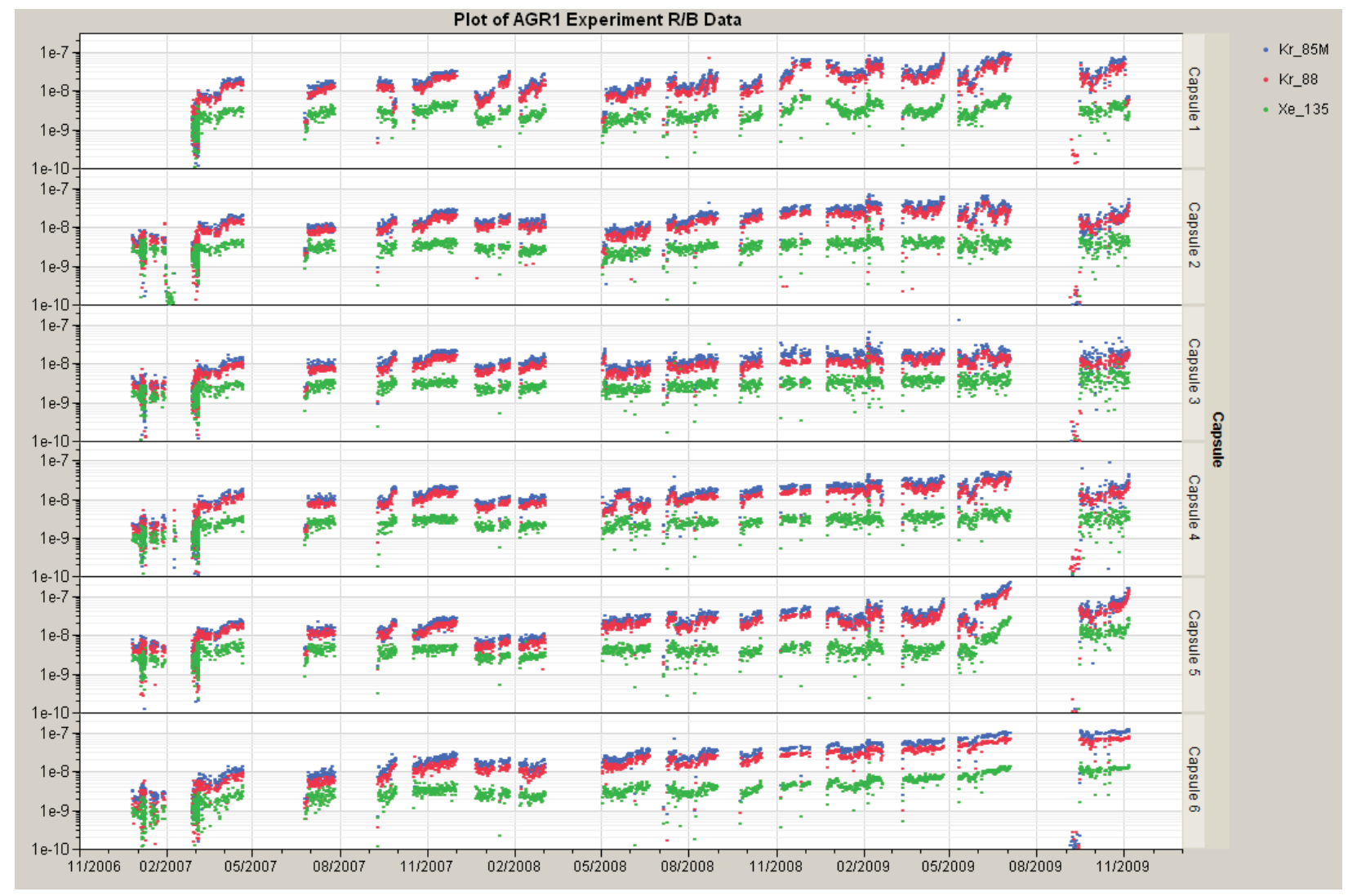

Figure 9. Example of an R/B data plot available on the NDMAS Web portal. 


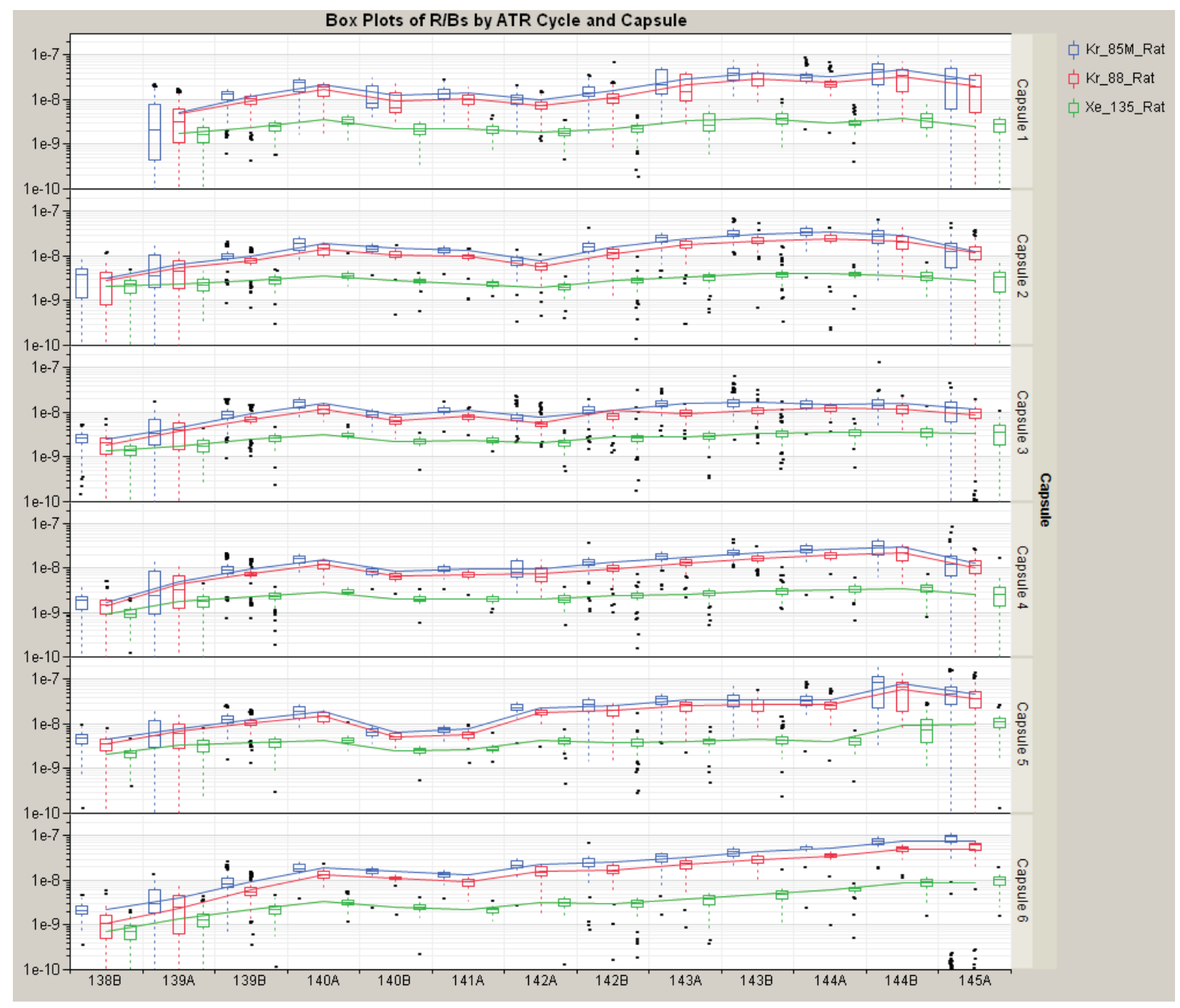

Figure 10. Example of release and R/B box plots available on the NDMAS Web portal. The boxes represent the $75 \%$ and $25 \%$ quartiles, with the center line representing the median R/B for each ATR cycle by capsule. 


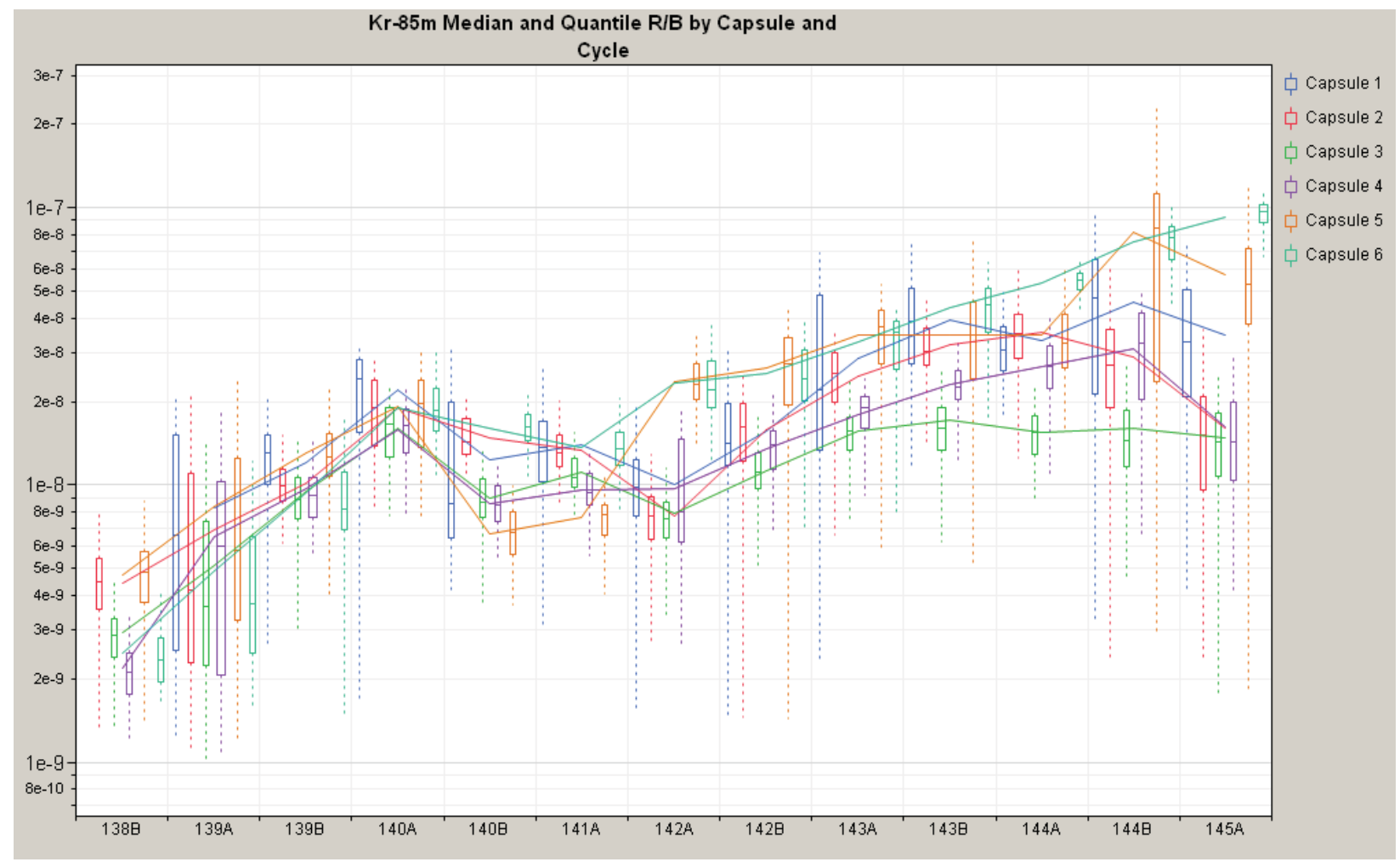

Figure 11. Example of FPMS box plots on the NDMAS Web portal showing Kr-85m R/B variations across capsules.

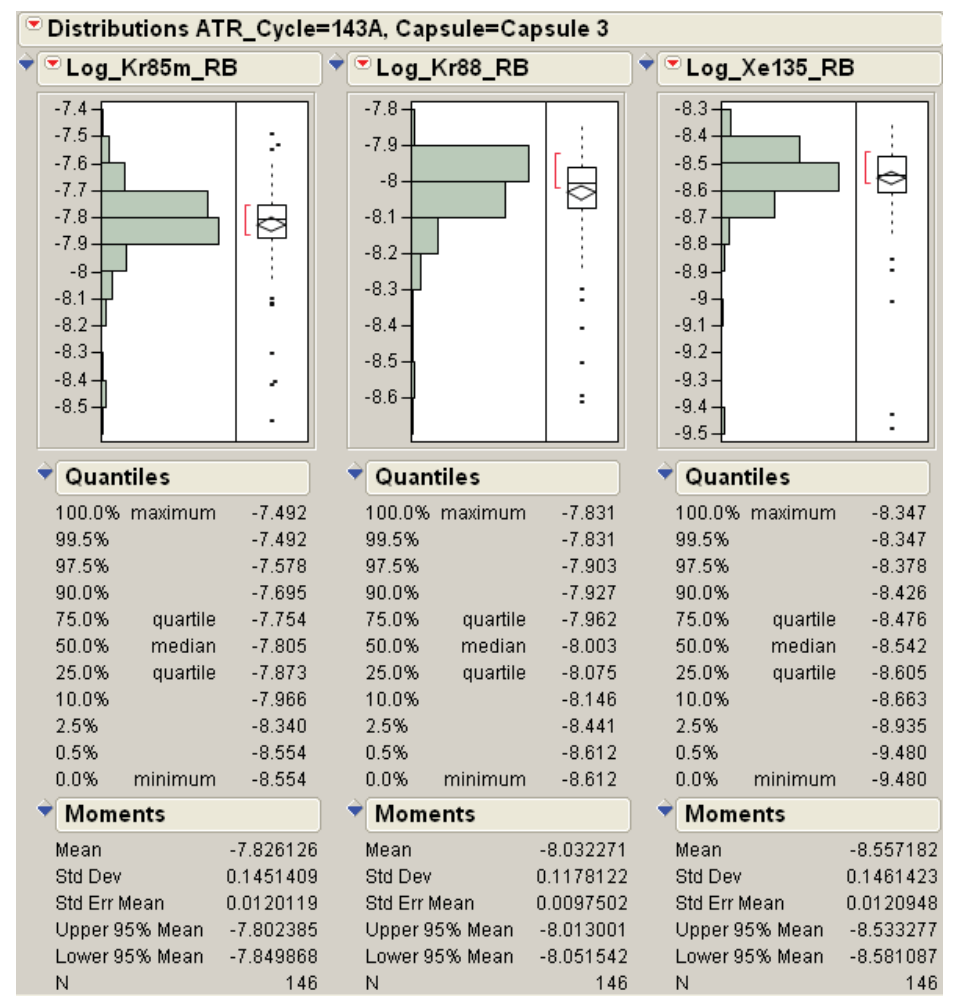

Figure 12. Example of [Log] R/B data distribution analysis available on the NDMAS Web portal. 


\subsection{FPMS Data Qualification Status}

The overall qualification status of all data stored in NDMAS is provided on the Qualification page of the NDMAS Web portal (https://sasweb.inl.gov). All of the FPMS data packages received have passed NDMAS capture testing with no identified errors, and the most current version of the data (January 2010 processing date) have been made available on the NDMAS web portal (FPMS Release and R/B Data page). Final qualification of these data will be formally documented within NDMAS after VHTR TDO QA approval of ECAR-907, which has been submitted by the FPMS technical staff and is currently under review. The FPMS R/B data may be revised in the future based on revisions or refinements in the modeled birth rate data used to calculate the R/B values. Revisions to the FPMS data such as these will be recorded with a source file date stamp in both the NDMAS database and Web portal to ensure users have the most recent data.

The ATR cycle data package start and end dates, number of records, and the most current FPMS source file dates stored in the NDMAS database are listed in Table 13.

Table 13. Current NDMAS database status of AGR-1 FPMS release and R/B data.

\begin{tabular}{|c|c|c|c|c|}
\hline $\begin{array}{c}\text { ATR Cycle Data } \\
\text { Package/ID } \\
\end{array}$ & Start Date/Time ${ }^{\mathrm{b}}$ & End Date/Time $^{\mathrm{b}}$ & $\begin{array}{l}\text { Number of } \\
\text { Records }\end{array}$ & Source File Dates ${ }^{\mathrm{d}}$ \\
\hline 138B/75 & 25DEC2006:00:13 & 10FEB2007:21:02 & 33,120 & $2 / 2 / 2010$ \\
\hline 139A/76 & 27FEB2007:01:45 & 23APR2007:08:40 & 77,712 & $2 / 2 / 2010$ \\
\hline 139B/77 & 23JUN2007:20:32 & 30SEP2007:05:50 & 57,312 & $1 / 31 / 2010$ \\
\hline $140 \mathrm{~A} / 78$ & 14OCT2007:19:48 & 02DEC2007:19:00 & 44,160 & $1 / 31 / 2010,2 / 1 / 2010$ \\
\hline 140B/79 & 21DEC2007:07:52 & 28JAN2008:23:56 & 34,272 & $2 / 2 / 2010$ \\
\hline $141 \mathrm{~A} / 80$ & 03FEB2008:05:45 & 02MAR2008:23:56 & 25,680 & $1 / 31 / 2010$ \\
\hline $142 \mathrm{~A} / 81$ & 01MAY2008:06:47 & 23JUN2008:08:19 & 47,376 & $1 / 31 / 2010$ \\
\hline 142B/82 & 03JUL2008:07:40 & 01SEP2008:04:00 & 55,440 & $1 / 30 / 2010$ \\
\hline $143 \mathrm{~A} / 83$ & 22SEP2008:07:24 & 10DEC2008:07:39 & 60,384 & $1 / 30 / 2010$ \\
\hline $143 \mathrm{~B} / 84$ & 21DEC2008:10:01 & 23FEB2009:14:57 & 64,128 & $1 / 30 / 2010$ \\
\hline $144 \mathrm{~A} / 85$ & 11MAR2009:16:34 & 26APR2009:20:34 & 41,712 & $1 / 30 / 2010$ \\
\hline 144B/86 & 09MAY2009:11:44 & 05JUL2009:23:17 & 52,416 & $1 / 30 / 2010$ \\
\hline \multirow[t]{2}{*}{$145 \mathrm{~A} / 88$} & 04SEP2009:08:03 & 09NOV2009:10:54 & 59,040 & $2 / 9 / 2010$ \\
\hline & & Total number of records $=$ & 652,752 & \\
\hline \multicolumn{5}{|c|}{$\begin{array}{l}\text { a. Database identification number for ATR cycle. } \\
\text { b. Source (raw data) date/time of first and last FPMS data record in that data package. } \\
\text { c. Single response variable records in the NDMAS (tall) database. } \\
\text { d. Latest source file date supplied by FPMS technical staff. }\end{array}$} \\
\hline
\end{tabular}

\subsection{FPMS Data Problems and Resolution}

Several revisions to the processed FPMS data have been generated by the FPMS technical staff after the initially-supplied preliminary data files were loaded into NDMAS, requiring that the NDMAS data be deleted, reloaded, and retested. These revisions were made because of a number of technical issues discovered after the initial data package submittal, including a discrepancy between the FPMS time scale (calendar days) and that used for modeled birthrates (effective power days), an error in the assumed reactor startup time for ATR Cycle 143A, and a problem on the spectral analysis of Kr- 88 . These issues led to the recent reprocessing of all FPMS release and R/B data files for all 13 AGR-1 cycles. The primary NDMAS issue with revisions to the FPMS data packages is how to best track and report these revisions within the NDMAS SQL database and on the Web portal, to ensure researchers are using the 
most current data. Old versions of FPMS data files are not currently maintained in the NDMAS SQL database, although they are maintained by the FPMS technical staff on the FSISC1 server. Data files available on the NDMAS Web portal ('FPMS Release and R/B Data' page) have the most recent source file date in the file name (e.g., Release_143A_01312010) and a text warning that all data files available for downloading are subject to revisions. 


\section{ATR OPERATING CONDITIONS DATA}

\subsection{Description of the Data Stream}

ATR operators collect data describing the configuration and state of the reactor at high frequency (sub-minute intervals), and some of those parameters are collected and stored in the NDMAS to facilitate various analyses of the AGR experimental data (e.g., thermocouple temperatures). At the beginning of the AGR-1 experiment, ATR operating conditions data were provided in miscellaneous files that contained data recorded at 1-hour intervals. Since April 25, 2008, ATR data have been provided to NDMAS in daily files with measurements at 1-minute intervals. The data provided and stored in NDMAS include the parameters summarized in Table 14.

Table 14. ATR operating conditions variables stored in NDMAS.

\begin{tabular}{|c|c|c|c|c|}
\hline Variable & $\begin{array}{c}\text { Captured } \\
\text { by } \\
\text { NDMAS }\end{array}$ & Description & $\begin{array}{c}\text { Example of } \\
\text { Raw Data } \\
\text { from 6-18- } \\
\text { 08:00:00:28 }\end{array}$ & Units \\
\hline TNRD & & Number of readings total & 1.00 & {$[-]$} \\
\hline FPRD & & Number of readings at full power & 1.00 & {$[-]$} \\
\hline FPTM & & Time at full power & 1.00 & {$[-]$} \\
\hline LCNW & $*$ & Current power for NW lobe & 22.94 & MW \\
\hline LCNE & $*$ & Current power for NE lobe & 18.05 & MW \\
\hline LC_C & $*$ & Current power for $\mathrm{C}$ lobe & 25.20 & MW \\
\hline LCSW & $*$ & Current power for SW lobe & 24.70 & MW \\
\hline LCSE & $*$ & Current power for SE lobe & 23.11 & MW \\
\hline LINW & & Integrated power for NW lobe & 1024.75 & MW \\
\hline LINE & & Integrated power for NE lobe & 801.69 & MW \\
\hline LI_C & & Integrated power for_C lobe & 1097.50 & MW \\
\hline LISW & & Integrated power for SW lobe & 1105.75 & MW \\
\hline LISE & & Integrated power for SE lobe & 1023.88 & MW \\
\hline N16R & & & 0.70 & \\
\hline TPQ1 & & Thermal power for quadrant 1 & 22.50 & MW \\
\hline TPQ2 & & Thermal power for quadrant 2 & 29.52 & MW \\
\hline TPQ3 & & Thermal power for quadrant 3 & 32.54 & MW \\
\hline TPQ4 & & Thermal power for quadrant 4 & 29.21 & MW \\
\hline TPSM & $*$ & Thermal power - sum & 113.77 & MW \\
\hline TPTO & $*$ & Thermal power - total & 112.88 & MW \\
\hline REG1 & $*$ & Position of regulating rod 1 & 28.65 & [in] \\
\hline REG2 & $*$ & Position of regulating rod 2 & 47.73 & [in] \\
\hline N3D4 & $*$ & Position of shim cylinders & 88.50 & [degrees] \\
\hline E1D2 & $*$ & Position of shim cylinders & 88.50 & [degrees] \\
\hline E3D4 & $*$ & Position of shim cylinders & 92.98 & [degrees] \\
\hline S1D2 & $*$ & Position of shim cylinders & 92.98 & [degrees] \\
\hline S3D4 & $*$ & Position of shim cylinders & 115.48 & [degrees] \\
\hline W1D2 & $*$ & Position of shim cylinders & 115.48 & [degrees] \\
\hline W3D4 & $*$ & Position of shim cylinders & 93.52 & [degrees] \\
\hline N1D2 & $*$ & Position of shim cylinders & 93.52 & [degrees] \\
\hline PCIT & $*$ & Reactor process water inlet temperature & 113.16 & {$\left[{ }^{\circ} \mathrm{F}\right]$} \\
\hline PCOT & & Reactor process water outlet temperature & 130.56 & {$\left[{ }^{\mathrm{o}} \mathrm{F}\right]$} \\
\hline
\end{tabular}


Table 14. (continued).

\begin{tabular}{|c|c|c|c|c|}
\hline Variable & $\begin{array}{c}\text { Captured } \\
\text { by } \\
\text { NDMAS }\end{array}$ & Description & $\begin{array}{l}\text { Example of } \\
\text { Raw Data from } \\
\text { 6-18- } \\
\text { 08:00:00:28 }\end{array}$ & Units \\
\hline RPWF & $*$ & Reactor process water flow & 43.70 & \\
\hline NERL & * & Position of NE neck shims (note 1) & 100000 & [-] \\
\hline SERL & $*$ & Position of SE neck shims (note 1) & 111300 & {$[-]$} \\
\hline SWRL & $*$ & Position of SW neck shims (note 1) & 111210 & {$[-]$} \\
\hline NWRL & * & Position of NW neck shims (note 1) & 111100 & {$[-]$} \\
\hline SROD & & Safety rod limit switch status (note 2) & & {$[-]$} \\
\hline RDAS & & Bit pattern sent by RDAS & 5.00 & {$[-]$} \\
\hline CSUM & & Checksum sent by RDAS & 13048.00 & {$[-]$} \\
\hline RDCD & & RDAS normal or abnormal (note 3 ) & 0.00 & {$[-]$} \\
\hline RDTD & & RDAS (DAC or DAN) transmitting data (note 4) & 1.00 & {$[-]$} \\
\hline N16D & $*$ & N16 system - normal or calibrate (note 5) & 0.00 & {$[-]$} \\
\hline OSCD & & $\begin{array}{l}\text { OSC indication - normal or error (bad drums are } \\
\text { indicated as zero) (note } 6 \text { ) }\end{array}$ & 0.00 & {$[-]$} \\
\hline WPMD & & Water power calculator in use-system 1 or 2 (note 7$)$ & 0.00 & {$[-]$} \\
\hline SPRD & & System power constraint & 0.00 & \\
\hline \multicolumn{5}{|c|}{ 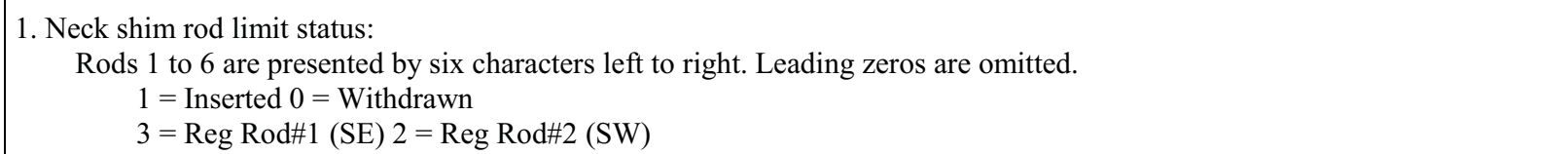 } \\
\hline \multicolumn{5}{|c|}{ 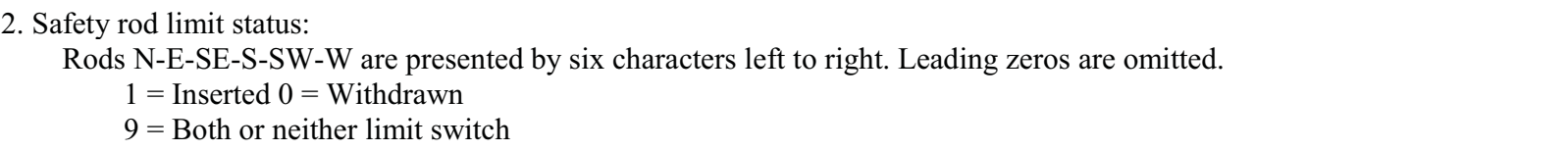 } \\
\hline \multicolumn{5}{|c|}{ 3. $\mathrm{RDCD}-\mathrm{RDAS}$ status $(0=$ Normal, $1=$ Abnormal $)$} \\
\hline \multicolumn{5}{|c|}{ 4. RDTD - RDAS system transmitting data $(0=\mathrm{DAC}, 1=\mathrm{DAN})$} \\
\hline \multicolumn{5}{|c|}{ 5. $\mathrm{N} 16 \mathrm{D}-\mathrm{N} 16$ system status $(0=$ Normal, $1=$ Calibrate $)$} \\
\hline \multicolumn{5}{|c|}{ 6. OSCD - Outer shim cylinders $(0=$ Normal, $1=$ Error $)$} \\
\hline \multicolumn{5}{|c|}{ 7. WPMD - Water power calculator $(0=$ System one, $1=$ System two $)$} \\
\hline
\end{tabular}

\subsection{ATR Data Processing within NDMAS}

Data processing and storage within NDMAS occurs via the following process shown in Figure 13. After raw ATR data files each covering one day of measurements are placed in folders on the FSISC1 server (AGR Data folder), those files are copied to an NDMAS server location devoted to raw data storage. A batch file is then run to update the NDMAS-maintained list of relevant file names in that directory, from which new file names will be processed. A SAS Enterprise Guide project entitled 'Update or build ATR dataset.egp' subsequently reads the new data files and assembles the data into a single SAS dataset, which represents the primary source for ATR data within NDMAS. Those data are averaged to 5-minute intervals to match the interval used for fuel irradiation monitoring and then stored in the NDMAS SQL database. Processing and storage in NDMAS occurs approximately once per week so that generally, numerous data files are processed and entered as a single package. 


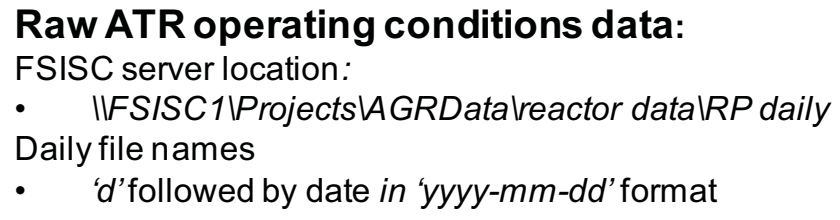

\section{ATR operating conditions processing steps:}

1) Copy new file folders from FSISC 1 server to:

- $\quad$ IISasngnpINGNPINGNP_DatalAGR-11reactor dataIRP daily

2) FROM NDMAS directory, 'Vault_PushPull'

1) Run batch file 'update.bat'

- Creates/updates the list of relevant file names to be processed

2) Run Enterprise Guide Project file 'Update or build ATR dataset.egp' to construct SAS dataset for new data

- Builds/appends all new data as SAS dataset

- Pushes new data to SQL_NDMAS (NDMAS vault)

- $\quad$ Checks that vault data is correctly entered

3) Update log file 'Irradiation capture-push instructions and log.doc'

4) Run Enterprise Guide Project file 'Vault tests.egp' and examine any failed data

5) Resolve failed data as necessary

6) Run Enterprise Guide Project file 'Combine_Irrad_ATR_FPMS.egp' to populate N_MART directory with new SAS data sets and cubes containing updated irradiation data

Figure 13. Raw ATR operating conditions data and processing steps.

Data processing includes the following error checks to ensure that the data are accurately captured:

- Dates are checked for proper syntax and chronology.

- Data are checked for duplicated measurement times with conflicting variable values.

- Completed SAS datasets are visually inspected and compared against the raw data files.

- A MST is assigned to each measurement that corrects for the switching between MST and MDT that occurs in the raw data. The switch to MDT leads to repeated measurement times with different variable values, while the switch to standard time leads to a gap in measurement times. The process of detecting the MDT shifts is not fully automated, because the changes are not always made at the correct date and time. The data is manually checked to find MDT shifts on dates/times that do not correspond with national shift time.

- The process of entering each data packages is recorded in an electronic log with appropriate notes about any problems or corrections encountered. After being entered into the NDMAS SQL database, each data package is compared to the SAS dataset from which it was built to ensure that the data were correctly stored. Results are stored in the electronic log "ATR capture-push instructions and log.doc."

- ATR parameters that reflect internal testing of the ATR system are used to identify potential problems with data from instruments or systems being tested. Lobe powers, for example, can fluctuate during calibration of the N16 system. The calibration system status indicators can thus be used to eliminate those data from datasets used for neutronics or other analyses. 


\subsection{ATR Data Qualification}

NDMAS conducts routine error testing on collected ATR data, but ATR operating conditions data are qualified by the quality control procedures of the ATR, not by the VHTR program or NDMAS. Technical Specifications for nuclear power plants define operational limits and conditions as a way to assure that the plant operates safely and in a manner that is consistent with the assumptions made in the plant safety analysis. Plant Technical Specifications are strictly followed during all stages of plant operation, and operating conditions monitoring data are collected from the plant to demonstrate compliance. BEA operates ATR for DOE under the Price-Anderson Amendments Act, which indemnifies DOE contractors from costs related to public liability for operating a nuclear facility. However, DOE is required to take enforcement actions against indemnified contractors for violations of nuclear safety requirements; that is, for operating the reactor outside the Technical Specifications. Data collected to monitor ATR operating conditions are used to demonstrate that the reactor is operating within the Technical Specifications. These data are collected within a well documented QA program compliant with NQA-1 requirements following well documented procedures. Audits, BEA management oversight, and DOE oversight ensure that data collected to demonstrate compliance with ATR Technical Specifications meet the QA and measurement requirements for monitoring ATR. NGNP accepts that the ATR data meet requirements based on the ATR data qualification program and does not take additional actions to qualify reactor operating conditions data. ATR data are read from raw files, captured into the NDMAS vault, and verified for capture.

\subsection{ATR Data Problems and Resolution}

During the early cycles of the AGR-1 experiment, NDMAS obtained ATR data from informal records of NGNP technical personnel, rather than directly from the ATR data recording system, and in several different file formats and with different parameter lists. To provide the highest-integrity record of ATR data, and to use, rather than reproduce, time-averaging and other data integration features of the ATR data collection system, it is recommended that NDMAS have a direct link to archived data of the ATR system and its qualification status records.

As with AGR-1 fuel irradiation monitoring data, ATR data are collected with a local date/time stamp that periodically shifts to MDT at times not always consistent with official times for those changes. To

provide a consistent time/record for all data sets, it is recommended that the ATR also maintain a MST or other standard-time date/time stamp for all records. 


\section{NEUTRONICS AND THERMAL SIMULATION DATA}

\subsection{Description of the Data Stream}

The neutronics and thermal (N\&T) data stream consists of daily and end-of-cycle (EOC) physics model simulation data of AGR-1 fuel performance and experimental component conditions. The response variables stored within NDMAS are grouped by simulation analysis type and data generator (i.e., modeler) as shown in Table 15. Two types of simulation data are stored for fission power, burnup, and fast neutron fluence: (1) EOC "as-run" calculations from Lillo and Chang (2009), and (2) daily calculations for each of 144 fuel compact cells. Other simulation data stored in NDMAS include daily heating rates for 22 capsule components, daily simulated fuel temperatures (averaged by compact, stack, and capsule), simulated thermocouple temperatures, and simulated radionuclide inventories (in progress).

Table 15. Neutronics and thermal simulation data being stored in NDMAS.

Variable Name Description

(1) End-of-Cycle MCNP and ORIGEN2 simulations for each compact cell (complete)

FD Fuel fission power density $\left(\mathrm{W} / \mathrm{cm}^{3}\right)$

FIMA ORIGEN2 Simulation Fuel burnup at cycle end date (\%FIMA)

FLUENCE MCNP Simulation Fast neutron fluence $(\mathrm{E}>0.18 \mathrm{MeV})$ at cycle end date $\left(\mathrm{x} 10^{20}\right.$ $\mathrm{n} / \mathrm{cm}^{2}$ )

(2) Daily MCNP and ORIGEN2 simulations for each compact cell (complete)

FD_D Total fission heat (MW)

FIMA_D Fuel burnup (\%FIMA)

FLUENCE_D Fast neutron fluence $\left(\mathrm{n} / \mathrm{cm}^{2}\right)$

(3) Daily simulations of 22 component heat rates for each capsule $\left(\mathrm{W} / \mathrm{cm}^{3}\right)$ (in progress)

(4) Daily ABACUS simulations of fuel temperatures averaged by compact, stack, and capsule; simulated thermocouple temperatures by capsule $\left({ }^{\circ} \mathrm{C}\right)$ (in progress)

\begin{tabular}{ll}
\hline SFT_AVE & Average fuel temperature \\
SFT_MIN & Minimum fuel temperature \\
SFT_MAX & Maximum fuel temperature \\
SFT_TA_AVE & Time-averaged Average fuel temperature \\
SFT_TA_MIN & Time-averaged Minimum fuel temperature \\
SFT_TA_MAX & Time-averaged Maximum fuel temperature \\
STC_[1,2,3,4,5] & Thermocouple temperature for capsules 1-5 \\
(5) End of cycle/experiment radionuclide inventories (in progress)
\end{tabular}

\subsection{Data Processing within NDMAS}

The following source data files have been received from the data generators (e.g., modelers):

- AGR1 Final Fuel AsRun Summary thru 145A.xls (1/22/2010 transmittal date) - includes EOC AsRun simulations for FD, FIMA, and FLUENCE

- IISterb_Hawkes_Heat $\backslash$ cycle \#\Heat $\backslash$ cycle \#_fiss.day\# - daily ASCII files for FD_D (1/26/2010 transmittal date).

- IISterbentz_FFluences\combo.[cycle \#].output - daily ASCII files for FLUENCE_D (2/3/2010 transmittal date). 
- Daily files for FIMA_D (Date/time)

- Master Output AGR DailyCalcs.xls (Date/time) - includes component heat rates [(3) in Table 15], simulated fuel and thermocouple temperatures [(4) in Table 15]

- AGR1_EFPD.xls - includes effective power days (2/4/2010 transmittal date).

All source data files are stored within NDMAS in the location: "\ISasngnp $\backslash N G N P \backslash N G N P \_D a t a \backslash A G R-$ $1 \backslash . "$ These files are captured and processed within NDMAS using the steps shown in Figure $1 \overline{4}$.

\section{N\&T Raw Data:}

- Raw data files received from data generators are stored in IISasngnp\NGNP\NGNP_Data\AGR-1\:

- $\quad$ AGR1 Final Fuel AsRun Summary thru 145A.xls

- Master Output AGR DailyCalcs.xls

- Sterbentz_FFluences $\backslash$ combo.[cycle \#].output

- Sterb_Hawkes_Heatlcycle \#Heat $\backslash$ cycle \#_fiss.day\#

- Use Matlā codes to extract and reorganize Sterbentz's FD_D and FLUENCE_D data from multiple raw data files, lineup Sterbentz's time steps with Hawkes's date and save the combined excel spreadsheets in $\backslash$ Sasngnp\NGNP\NGNP_Data\AGR-1 $\backslash$ before importing into SAS data sets:

- AGR_Temp_Fission_Gamma.xls for FD_D

- AGR_FF_STERB_day.xls for FLUENCE__D

\section{N\&T SAS Data Sets:}

\ISasngnp \NGNP\NDMAS Version 1.1\Neutronics_Thermal\:

- Upload Neut_Temp to data_table.egp: SAS processing

- Import data from excel spreadsheets

- Transpose data to make a tall table with one response value per line

- Assign predefined response variable name, label, and description to each response value

- Define component name for each response value (AGR-1 experiment, Capsule, Stack, Compact, or Compact cell) according to predefined component list "usv_Neutronics_Components"

- Get component ID based on its name from the list "Exp_comp"

- Get response variable ID based on response variable name from the list "Response_Var_Attrib"

- The following intermediate SAS datasets are stored in the N_Depot library for inspection before pushing into the vault:

○ AGR1_NEUTRONIC_DATA for EOC As-Run simulations for FD, FIMA, and FLUENCE

- AGR1_FASTFLUENCE_STERB for daily FLUENCE_D

- AGR1SIM_FUEL_HEAT for daily FD_D

- AGR1_SIM_TEMPERATURE for simulated fuel and thermocouple temperatures

○ AGR1_EFPD for effective power days

Figure 14. N\&T data processing schematic.

Most of N\&T data sets represent model predictions at each of 144 fuel compact cells (or modeling "nodes") counting from the top down with cells 1-48 in Stack 1, 49-96 in Stack 3, and 97-144 in Stack 2. The cell numbering scheme relative to the physical compact loading "level" within a capsule is shown in Figure 15. The SAS E-Guide processing listed in Figure 14 links the model output cell numbers to the 
fuel compact name, capsule, stack, and loading level so that the modeling results can be linked to individual compact fabrication specifications as listed in Appendix A.

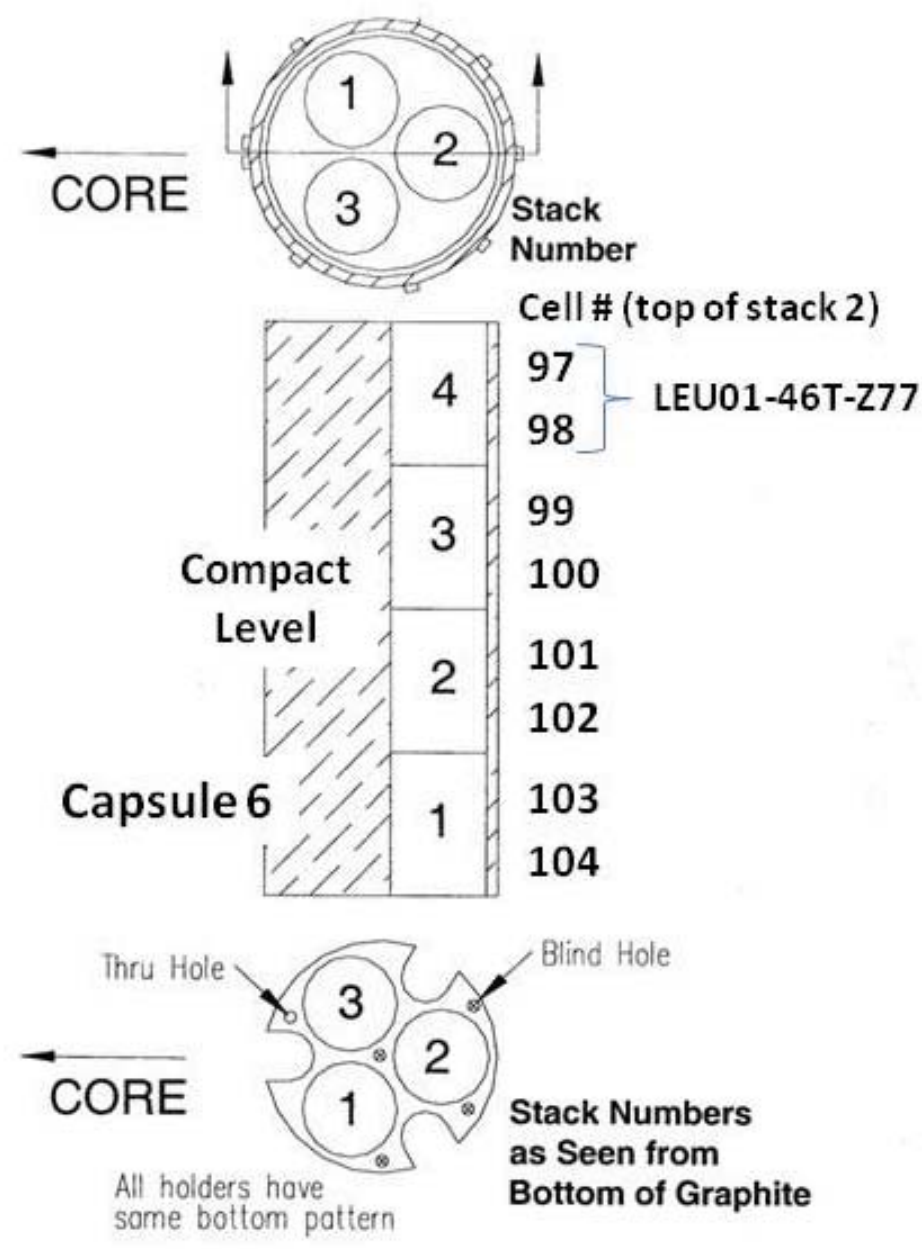

Figure 15. NDMAS links the modeling cell number to compact stack, loading levels, and lot names (shown here for top of stack 2).

\subsection{Data Qualification}

The neutronics and thermal data are physics model predictions that cannot be rigorously validated (except perhaps through post irradiation examination of the modeled components). Therefore, for this data stream, NDMAS data qualification consists only of capture testing to ensure the data stored in the database are identical to the raw data files loaded into the database, and documentation of the modeling analyses in approved reports (e.g., ECARs) produced by the data generators (e.g., Lilo and Chang 2009). Model reports for most of these data are still in progress.

\section{REFERENCES}

Ambrosek, R. G., 2007, “AGR-1 As-Run Thermal Evaluations - Cycle 138B, 139A,B,” ECAR-102, Draft December 4, 2007.

ASME, 2000, "Quality Assurance Requirements for Nuclear Facility Applications," Nuclear Quality Assurance (NQA-1), ASME International, New York, NY. 
Barnes, C. M., 2005, “Disposition of BWXT Kernel Lot G73D-20-69302,” EDF-5796, Rev. 0, $04 / 11 / 2005$.

Barnes, C. M., 2006, “AGR-1 Fuel Product Specification and Characterization Guidance,” EDF-4380, Rev. 8, 04/21/2006.

BWXT Nuclear Products Division, 2005, "Industrial Fuel Fabrication and Development Lot G73D-2069302,” AGR-1 Fuel Kernel Data Certification Package, March 11, 2005.

BWXT, “Operating Plan for Industrial Fuel Development," OP-1014307, Rev 00, BWX Technologies Inc., Lynchburg, Virginia.

Drigert, M. W., D. M. Scates, J. B. Walter, 2009, "Users' Guide for the Fission Product Monitoring System," GDE-503, Rev 0, 04/16/09.

Ebner, M. A., "Composition Distribution of Fuel Kernels in LEU Composite 69302," EDF-5723, Rev. 0, 04/12/2005.

Einerson, J. J., 2006, “Statistical Sampling Plan for AGR Fuel Materials,” EDF 4542, Rev. 7, 02/01/2006.

Hartwell, J. K., J. B. Walter, D. M. Scates, M. W. Drigert, 2007, Determination of the AGR-1 Capsule to FPMS Spectrometer Transport Volumes from Leadout Flow Test Data, INL/EXT-07-12494, May 2007.

Hull, Laurence C., AGC-1 Data Qualification Interim Report, INL/EXT-09-16484, Idaho National Laboratory, August 2009.

Hunn, J. D. and R. A. Lowden, 2006a, “AGR-1 Variant 1 Fuel Final Data Package,” Rev. 1, September 20, 2006.

Hunn, J. D. and R. A. Lowden, 2006b, “AGR-1 Variant 2 Fuel Final Data Package,” Rev. 1, September 20, 2006.

Hunn, J. D. and R. A. Lowden, 2006c, “AGR-1 Variant 3 Fuel Final Data Package,” Rev. 1, September 20, 2006.

Hunn, J. D. and R. A. Lowden, 2009, “AGR-1 Baseline Fuel Final Data Package,” Rev. 2, March 30, 2009.

INL, 2008, FY 2008 Advanced Test Reactor National Scientific User Facility Users' Guide, INL/EXT-07-13577, Idaho National Laboratory, Idaho Falls, ID, 38 p.

INL, 2009a, "Very High Temperature Reactor Program Data Management and Analysis Plan," PLN-2709 Rev 1, Idaho National Laboratory, Idaho Falls, ID.

INL, 2009b, "Very High Temperature Gas Reactor Technology Development Office Quality Assurance Program Plan," PLN-2690, Rev 3, 07/22/2009.

INL ICARE NCR-42791, 2008, “ATR Experiment Data Collection System and Resulting Data,” May 28, 2008.

Lilo, M.A. and Chang, G.S., 2009, "Neutronics Projection Analysis to Support the End of Irradiation Determination for the AGR-1 Experiment," ECAR-515, February 2009.

Maki, J. T., 2007, AGR-1 Irradiation Experiment Test Plan, INL/EXT-05-00593, Rev 2, March 2007.

Maki, J. T., 2007b, AGR-1 As-Run Analysis Status For FY-07, INL/EXT-07-13630, Rev 0, December 2007.

ORNL, 2006, "Quality Assurance Plan for The Advanced Gas Reactor Fuel Development and Qualification Program at the Oak Ridge National Laboratory," QAP-ORNL-AGR-01, Rev. 5, Oak Ridge National Laboratory, July 2006. 
Palmer, A. J., 2006, “Thermocouple recommendations for the AGR-1 test,” EDF-6809, Rev. 0, May 2006.

Scates, D. M., 2010, "Release to Birth Ratios for AGR-1 Operating Cycles 138B through 145A," ECAR-907, February 2010.

Scates, D. M. and J. K. Hartwell, 2006, "Fission Product Monitoring System Operability Test Plan for the AGR-1 Experiment: Phase II,” PLN-2350, December 2006.

West, P. B., G. A. Marts, E. W. Killian, J. K. Hartwell, and S. B. Grover, 2005, "Temperature Control and Off Gas Monitoring Systems for Advanced Gas Reactor Experiment AGR-1,” TFR-248, Rev. 1, March 2005. 
Appendix A

NDMAS Database Join for Compacts and Modeling Cells 


\section{Appendix A \\ NDMAS Database Join for Compacts and Modeling Cells}

\begin{tabular}{|c|c|c|c|c|c|}
\hline Stack_Name & Compact_Name & Type & Level & Compact-Cell & Cell \\
\hline Capsule_6_Stack_1 & LEU01-46T-Z53 & Baseline & 4 & LEU01-46T-Z53-N1 & 1 \\
\hline Capsule_6_Stack_1 & LEU01-46T-Z53 & Baseline & 4 & LEU01-46T-Z53-N2 & 2 \\
\hline Capsule_6_Stack_1 & LEU01-46T-Z49 & Baseline & 3 & LEU01-46T-Z49-N1 & 3 \\
\hline Capsule_6_Stack_1 & LEU01-46T-Z49 & Baseline & 3 & LEU01-46T-Z49-N2 & 4 \\
\hline Capsule_6_Stack_1 & LEU01-46T-Z33 & Baseline & 2 & LEU01-46T-Z33-N1 & 5 \\
\hline Capsule_6_Stack_1 & LEU01-46T-Z33 & Baseline & 2 & LEU01-46T-Z33-N2 & 6 \\
\hline Capsule_6_Stack_1 & LEU01-46T-Z32 & Baseline & 1 & LEU01-46T-Z32-N1 & 7 \\
\hline Capsule_6_Stack_1 & LEU01-46T-Z32 & Baseline & 1 & LEU01-46T-Z32-N2 & 8 \\
\hline Capsule_5_Stack_1 & LEU01-47T-Z79 & Variant1 & 4 & LEU01-47T-Z79-N1 & 9 \\
\hline Capsule_5_Stack_1 & LEU01-47T-Z79 & Variant1 & 4 & LEU01-47T-Z79-N2 & 10 \\
\hline Capsule_5_Stack_1 & LEU01-47T-Z23 & Variant1 & 3 & LEU01-47T-Z23-N1 & 11 \\
\hline Capsule_5_Stack_1 & LEU01-47T-Z23 & Variant1 & 3 & LEU01-47T-Z23-N2 & 12 \\
\hline Capsule_5_Stack_1 & LEU01-47T-Z13 & Variant1 & 2 & LEU01-47T-Z13-N1 & 13 \\
\hline Capsule_5_Stack_1 & LEU01-47T-Z13 & Variant1 & 2 & LEU01-47T-Z13-N2 & 14 \\
\hline Capsule_5_Stack_1 & LEU01-47T-Z08 & Variant1 & 1 & LEU01-47T-Z08-N1 & 15 \\
\hline Capsule_5_Stack_1 & LEU01-47T-Z08 & Variant1 & 1 & LEU01-47T-Z08-N2 & 16 \\
\hline Capsule_4_Stack_1 & LEU01-49T-Z72 & Variant3 & 4 & LEU01-49T-Z72-N1 & 17 \\
\hline Capsule_4_Stack_1 & LEU01-49T-Z72 & Variant3 & 4 & LEU01-49T-Z72-N2 & 18 \\
\hline Capsule_4_Stack_1 & LEU01-49T-Z68 & Variant3 & 3 & LEU01-49T-Z68-N1 & 19 \\
\hline Capsule_4_Stack_1 & LEU01-49T-Z68 & Variant3 & 3 & LEU01-49T-Z68-N2 & 20 \\
\hline Capsule_4_Stack_1 & LEU01-49T-Z64 & Variant3 & 2 & LEU01-49T-Z64-N1 & 21 \\
\hline Capsule_4_Stack_1 & LEU01-49T-Z64 & Variant3 & 2 & LEU01-49T-Z64-N2 & 22 \\
\hline Capsule_4_Stack_1 & LEU01-49T-Z58 & Variant3 & 1 & LEU01-49T-Z58-N1 & 23 \\
\hline Capsule_4_Stack_1 & LEU01-49T-Z58 & Variant3 & 1 & LEU01-49T-Z58-N2 & 24 \\
\hline Capsule_3_Stack_1 & LEU01-46T-Z65 & Baseline & 4 & LEU01-46T-Z65-N1 & 25 \\
\hline Capsule_3_Stack_1 & LEU01-46T-Z65 & Baseline & 4 & LEU01-46T-Z65-N2 & 26 \\
\hline Capsule_3_Stack_1 & LEU01-46T-Z39 & Baseline & 3 & LEU01-46T-Z39-N1 & 27 \\
\hline Capsule_3_Stack_1 & LEU01-46T-Z39 & Baseline & 3 & LEU01-46T-Z39-N2 & 28 \\
\hline Capsule_3_Stack_1 & LEU01-46T-Z24 & Baseline & 2 & LEU01-46T-Z24-N1 & 29 \\
\hline Capsule_3_Stack_1 & LEU01-46T-Z24 & Baseline & 2 & LEU01-46T-Z24-N2 & 30 \\
\hline Capsule_3_Stack_1 & LEU01-46T-Z23 & Baseline & 1 & LEU01-46T-Z23-N1 & 31 \\
\hline Capsule_3_Stack_1 & LEU01-46T-Z23 & Baseline & 1 & LEU01-46T-Z23-N2 & 32 \\
\hline Capsule_2_Stack_1 & LEU01-48T-Z56 & Variant2 & 4 & LEU01-48T-Z56-N1 & 33 \\
\hline Capsule_2_Stack_1 & LEU01-48T-Z56 & Variant2 & 4 & LEU01-48T-Z56-N2 & 34 \\
\hline Capsule_2_Stack_1 & LEU01-48T-Z03 & Variant2 & 3 & LEU01-48T-Z03-N1 & 35 \\
\hline Capsule_2_Stack_1 & LEU01-48T-Z03 & Variant2 & 3 & LEU01-48T-Z03-N2 & 36 \\
\hline Capsule_2_Stack_1 & LEU01-48T-Z43 & Variant2 & 2 & LEU01-48T-Z43-N1 & 37 \\
\hline Capsule_2_Stack_1 & LEU01-48T-Z43 & Variant2 & 2 & LEU01-48T-Z43-N2 & 38 \\
\hline
\end{tabular}




\begin{tabular}{|c|c|c|c|c|c|}
\hline Stack_Name & Compact_Name & Type & Level & Compact-Cell & Cell \\
\hline Capsule_2_Stack_1 & LEU01-48T-Z27 & Variant2 & 1 & LEU01-48T-Z27-N1 & 39 \\
\hline Capsule_2_Stack_1 & LEU01-48T-Z27 & Variant2 & 1 & LEU01-48T-Z27-N2 & 40 \\
\hline Capsule_1_Stack_1 & LEU01-49T-Z77 & Variant3 & 4 & LEU01-49T-Z77-N1 & 41 \\
\hline Capsule_1_Stack_1 & LEU01-49T-Z77 & Variant3 & 4 & LEU01-49T-Z77-N2 & 42 \\
\hline Capsule_1_Stack_1 & LEU01-49T-Z02 & Variant3 & 3 & LEU01-49T-ZO2-N1 & 43 \\
\hline Capsule_1_Stack_1 & LEU01-49T-ZO2 & Variant3 & 3 & LEU01-49T-Z02-N2 & 44 \\
\hline Capsule_1_Stack_1 & LEU01-49T-Z54 & Variant3 & 2 & LEU01-49T-Z54-N1 & 45 \\
\hline Capsule_1_Stack_1 & LEU01-49T-Z54 & Variant3 & 2 & LEU01-49T-Z54-N2 & 46 \\
\hline Capsule_1_Stack_1 & LEU01-49T-Z69 & Variant3 & 1 & LEU01-49T-Z69-N1 & 47 \\
\hline Capsule_1_Stack_1 & LEU01-49T-Z69 & Variant3 & 1 & LEU01-49T-Z69-N2 & 48 \\
\hline Capsule_6_Stack_3 & LEU01-46T-Z76 & Baseline & 4 & LEU01-46T-Z76-N1 & 49 \\
\hline Capsule_6_Stack_3 & LEU01-46T-Z76 & Baseline & 4 & LEU01-46T-Z76-N2 & 50 \\
\hline Capsule_6_Stack_3 & LEU01-46T-Z34 & Baseline & 3 & LEU01-46T-Z34-N1 & 51 \\
\hline Capsule_6_Stack_3 & LEU01-46T-Z34 & Baseline & 3 & LEU01-46T-Z34-N2 & 52 \\
\hline Capsule_6_Stack_3 & LEU01-46T-Z30 & Baseline & 2 & LEU01-46T-Z30-N1 & 53 \\
\hline Capsule_6_Stack_3 & LEU01-46T-Z30 & Baseline & 2 & LEU01-46T-Z30-N2 & 54 \\
\hline Capsule_6_Stack_3 & LEU01-46T-Z14 & Baseline & 1 & LEU01-46T-Z14-N1 & 55 \\
\hline Capsule_6_Stack_3 & LEU01-46T-Z14 & Baseline & 1 & LEU01-46T-Z14-N2 & 56 \\
\hline Capsule_5_Stack_3 & LEU01-47T-Z72 & Variant1 & 4 & LEU01-47T-Z72-N1 & 57 \\
\hline Capsule_5_Stack_3 & LEU01-47T-Z72 & Variant1 & 4 & LEU01-47T-Z72-N2 & 58 \\
\hline Capsule_5_Stack_3 & LEU01-47T-Z71 & Variant1 & 3 & LEU01-47T-Z71-N1 & 59 \\
\hline Capsule_5_Stack_3 & LEU01-47T-Z71 & Variant1 & 3 & LEU01-47T-Z71-N2 & 60 \\
\hline Capsule_5_Stack_3 & LEU01-47T-Z65 & Variant1 & 2 & LEU01-47T-Z65-N1 & 61 \\
\hline Capsule_5_Stack_3 & LEU01-47T-Z65 & Variant1 & 2 & LEU01-47T-Z65-N2 & 62 \\
\hline Capsule_5_Stack_3 & LEU01-47T-Z55 & Variant1 & 1 & LEU01-47T-Z55-N1 & 63 \\
\hline Capsule_5_Stack_3 & LEU01-47T-Z55 & Variant1 & 1 & LEU01-47T-Z55-N2 & 64 \\
\hline Capsule_4_Stack_3 & LEU01-49T-Z20 & Variant3 & 4 & LEU01-49T-Z20-N1 & 65 \\
\hline Capsule_4_Stack_3 & LEU01-49T-Z20 & Variant3 & 4 & LEU01-49T-Z20-N2 & 66 \\
\hline Capsule_4_Stack_3 & LEU01-49T-Z36 & Variant3 & 3 & LEU01-49T-Z36-N1 & 67 \\
\hline Capsule_4_Stack_3 & LEU01-49T-Z36 & Variant3 & 3 & LEU01-49T-Z36-N2 & 68 \\
\hline Capsule_4_Stack_3 & LEU01-49T-Z17 & Variant3 & 2 & LEU01-49T-Z17-N1 & 69 \\
\hline Capsule_4_Stack_3 & LEU01-49T-Z17 & Variant3 & 2 & LEU01-49T-Z17-N2 & 70 \\
\hline Capsule_4_Stack_3 & LEU01-49T-Z14 & Variant3 & 1 & LEU01-49T-Z14-N1 & 71 \\
\hline Capsule_4_Stack_3 & LEU01-49T-Z14 & Variant3 & 1 & LEU01-49T-Z14-N2 & 72 \\
\hline Capsule_3_Stack_3 & LEU01-46T-Z79 & Baseline & 4 & LEU01-46T-Z79-N1 & 73 \\
\hline Capsule_3_Stack_3 & LEU01-46T-Z79 & Baseline & 4 & LEU01-46T-Z79-N2 & 74 \\
\hline Capsule_3_Stack_3 & LEU01-46T-Z56 & Baseline & 3 & LEU01-46T-Z56-N1 & 75 \\
\hline Capsule_3_Stack_3 & LEU01-46T-Z56 & Baseline & 3 & LEU01-46T-Z56-N2 & 76 \\
\hline Capsule_3_Stack_3 & LEU01-46T-Z55 & Baseline & 2 & LEU01-46T-Z55-N1 & 77 \\
\hline Capsule_3_Stack_3 & LEU01-46T-Z55 & Baseline & 2 & LEU01-46T-Z55-N2 & 78 \\
\hline Capsule_3_Stack_3 & LEU01-46T-Z17 & Baseline & 1 & LEU01-46T-Z17-N1 & 79 \\
\hline Capsule_3_Stack_3 & LEU01-46T-Z17 & Baseline & 1 & LEU01-46T-Z17-N2 & 80 \\
\hline
\end{tabular}




\begin{tabular}{|c|c|c|c|c|c|}
\hline Stack_Name & Compact_Name & Type & Level & Compact-Cell & Cell \\
\hline Capsule_2_Stack_3 & LEU01-48T-Z67 & Variant2 & 4 & LEU01-48T-Z67-N1 & 81 \\
\hline Capsule_2_Stack_3 & LEU01-48T-Z67 & Variant2 & 4 & LEU01-48T-Z67-N2 & 82 \\
\hline Capsule_2_Stack_3 & LEU01-48T-Z66 & Variant2 & 3 & LEU01-48T-Z66-N1 & 83 \\
\hline Capsule_2_Stack_3 & LEU01-48T-Z66 & Variant2 & 3 & LEU01-48T-Z66-N2 & 84 \\
\hline Capsule_2_Stack_3 & LEU01-48T-Z63 & Variant2 & 2 & LEU01-48T-Z63-N1 & 85 \\
\hline Capsule_2_Stack_3 & LEU01-48T-Z63 & Variant2 & 2 & LEU01-48T-Z63-N2 & 86 \\
\hline Capsule_2_Stack_3 & LEU01-48T-Z58 & Variant2 & 1 & LEU01-48T-Z58-N1 & 87 \\
\hline Capsule_2_Stack_3 & LEU01-48T-Z58 & Variant2 & 1 & LEU01-48T-Z58-N2 & 88 \\
\hline Capsule_1_Stack_3 & LEU01-49T-Z52 & Variant3 & 4 & LEU01-49T-Z52-N1 & 89 \\
\hline Capsule_1_Stack_3 & LEU01-49T-Z52 & Variant3 & 4 & LEU01-49T-Z52-N2 & 90 \\
\hline Capsule_1_Stack_3 & LEU01-49T-Z49 & Variant3 & 3 & LEU01-49T-Z49-N1 & 91 \\
\hline Capsule_1_Stack_3 & LEU01-49T-Z49 & Variant3 & 3 & LEU01-49T-Z49-N2 & 92 \\
\hline Capsule_1_Stack_3 & LEU01-49T-Z34 & Variant3 & 2 & LEU01-49T-Z34-N1 & 93 \\
\hline Capsule_1_Stack_3 & LEU01-49T-Z34 & Variant3 & 2 & LEU01-49T-Z34-N2 & 94 \\
\hline Capsule_1_Stack_3 & LEU01-49T-Z33 & Variant3 & 1 & LEU01-49T-Z33-N1 & 95 \\
\hline Capsule_1_Stack_3 & LEU01-49T-Z33 & Variant3 & 1 & LEU01-49T-Z33-N2 & 96 \\
\hline Capsule_6_Stack_2 & LEU01-46T-Z77 & Baseline & 4 & LEU01-46T-Z77-N1 & 97 \\
\hline Capsule_6_Stack_2 & LEU01-46T-Z77 & Baseline & 4 & LEU01-46T-Z77-N2 & 98 \\
\hline Capsule_6_Stack_2 & LEU01-46T-Z69 & Baseline & 3 & LEU01-46T-Z69-N1 & 99 \\
\hline Capsule_6_Stack_2 & LEU01-46T-Z69 & Baseline & 3 & LEU01-46T-Z69-N2 & 100 \\
\hline Capsule_6_Stack_2 & LEU01-46T-Z09 & Baseline & 2 & LEU01-46T-Z09-N1 & 101 \\
\hline Capsule_6_Stack_2 & LEU01-46T-Z09 & Baseline & 2 & LEU01-46T-Z09-N2 & 102 \\
\hline Capsule_6_Stack_2 & LEU01-46T-Z07 & Baseline & 1 & LEU01-46T-Z07-N1 & 103 \\
\hline Capsule_6_Stack_2 & LEU01-46T-Z07 & Baseline & 1 & LEU01-46T-Z07-N2 & 104 \\
\hline Capsule_5_Stack_2 & LEU01-47T-Z74 & Variant1 & 4 & LEU01-47T-Z74-N1 & 105 \\
\hline Capsule_5_Stack_2 & LEU01-47T-Z74 & Variant1 & 4 & LEU01-47T-Z74-N2 & 106 \\
\hline Capsule_5_Stack_2 & LEU01-47T-Z66 & Variant1 & 3 & LEU01-47T-Z66-N1 & 107 \\
\hline Capsule_5_Stack_2 & LEU01-47T-Z66 & Variant1 & 3 & LEU01-47T-Z66-N2 & 108 \\
\hline Capsule_5_Stack_2 & LEU01-47T-Z56 & Variant1 & 2 & LEU01-47T-Z56-N1 & 109 \\
\hline Capsule_5_Stack_2 & LEU01-47T-Z56 & Variant1 & 2 & LEU01-47T-Z56-N2 & 110 \\
\hline Capsule_5_Stack_2 & LEU01-47T-Z07 & Variant1 & 1 & LEU01-47T-Z07-N1 & 111 \\
\hline Capsule_5_Stack_2 & LEU01-47T-Z07 & Variant1 & 1 & LEU01-47T-Z07-N2 & 112 \\
\hline Capsule_4_Stack_2 & LEU01-49T-Z38 & Variant3 & 4 & LEU01-49T-Z38-N1 & 113 \\
\hline Capsule_4_Stack_2 & LEU01-49T-Z38 & Variant3 & 4 & LEU01-49T-Z38-N2 & 114 \\
\hline Capsule_4_Stack_2 & LEU01-49T-Z44 & Variant3 & 3 & LEU01-49T-Z44-N1 & 115 \\
\hline Capsule_4_Stack_2 & LEU01-49T-Z44 & Variant3 & 3 & LEU01-49T-Z44-N2 & 116 \\
\hline Capsule_4_Stack_2 & LEU01-49T-Z76 & Variant3 & 2 & LEU01-49T-Z76-N1 & 117 \\
\hline Capsule_4_Stack_2 & LEU01-49T-Z76 & Variant3 & 2 & LEU01-49T-Z76-N2 & 118 \\
\hline Capsule_4_Stack_2 & LEU01-49T-Z73 & Variant3 & 1 & LEU01-49T-Z73-N1 & 119 \\
\hline Capsule_4_Stack_2 & LEU01-49T-Z73 & Variant3 & 1 & LEU01-49T-Z73-N2 & 120 \\
\hline Capsule_3_Stack_2 & LEU01-46T-Z44 & Baseline & 4 & LEU01-46T-Z44-N1 & 121 \\
\hline Capsule_3_Stack_2 & LEU01-46T-Z44 & Baseline & 4 & LEU01-46T-Z44-N2 & 122 \\
\hline
\end{tabular}




\begin{tabular}{|cclccc|}
\hline Stack_Name & Compact_Name & Type & Level & Compact-Cell & Cell \\
\hline Capsule_3_Stack_2 & LEU01-46T-Z36 & Baseline & 3 & LEU01-46T-Z36-N1 & 123 \\
\hline Capsule_3_Stack_2 & LEU01-46T-Z36 & Baseline & 3 & LEU01-46T-Z36-N2 & 124 \\
\hline Capsule_3_Stack_2 & LEU01-46T-Z15 & Baseline & 2 & LEU01-46T-Z15-N1 & 125 \\
\hline Capsule_3_Stack_2 & LEU01-46T-Z15 & Baseline & 2 & LEU01-46T-Z15-N2 & 126 \\
\hline Capsule_3_Stack_2 & LEU01-46T-Z12 & Baseline & 1 & LEU01-46T-Z12-N1 & 127 \\
\hline Capsule_3_Stack_2 & LEU01-46T-Z12 & Baseline & 1 & LEU01-46T-Z12-N2 & 128 \\
\hline Capsule_2_Stack_2 & LEU01-48T-Z44 & Variant2 & 4 & LEU01-48T-Z44-N1 & 129 \\
\hline Capsule_2_Stack_2 & LEU01-48T-Z44 & Variant2 & 4 & LEU01-48T-Z44-N2 & 130 \\
\hline Capsule_2_Stack_2 & LEU01-48T-Z38 & Variant2 & 3 & LEU01-48T-Z38-N1 & 131 \\
\hline Capsule_2_Stack_2 & LEU01-48T-Z38 & Variant2 & 3 & LEU01-48T-Z38-N2 & 132 \\
\hline Capsule_2_Stack_2 & LEU01-48T-Z20 & Variant2 & 2 & LEU01-48T-Z20-N1 & 133 \\
\hline Capsule_2_Stack_2 & LEU01-48T-Z20 & Variant2 & 2 & LEU01-48T-Z20-N2 & 134 \\
\hline Capsule_2_Stack_2 & LEU01-48T-Z15 & Variant2 & 1 & LEU01-48T-Z15-N1 & 135 \\
\hline Capsule_2_Stack_2 & LEU01-48T-Z15 & Variant2 & 1 & LEU01-48T-Z15-N2 & 136 \\
\hline Capsule_1_Stack_2 & LEU01-49T-Z28 & Variant3 & 4 & LEU01-49T-Z28-N1 & 137 \\
\hline Capsule_1_Stack_2 & LEU01-49T-Z28 & Variant3 & 4 & LEU01-49T-Z28-N2 & 138 \\
\hline Capsule_1_Stack_2 & LEU01-49T-Z19 & Variant3 & 3 & LEU01-49T-Z19-N1 & 139 \\
\hline Capsule_1_Stack_2 & LEU01-49T-Z19 & Variant3 & 3 & LEU01-49T-Z19-N2 & 140 \\
\hline Capsule_1_Stack_2 & LEU01-49T-Z03 & Variant3 & 2 & LEU01-49T-Z03-N1 & 141 \\
\hline Capsule_1_Stack_2 & LEU01-49T-Z03 & Variant3 & 2 & LEU01-49T-Z03-N2 & 142 \\
\hline Capsule_1_Stack_2 & LEU01-49T-Z01 & Variant3 & 1 & LEU01-49T-Z01-N1 & 143 \\
\hline Capsule_1_Stack_2 & LEU01-49T-Z01 & Variant3 & 1 & LEU01-49T-Z01-N2 & 144 \\
\hline
\end{tabular}

